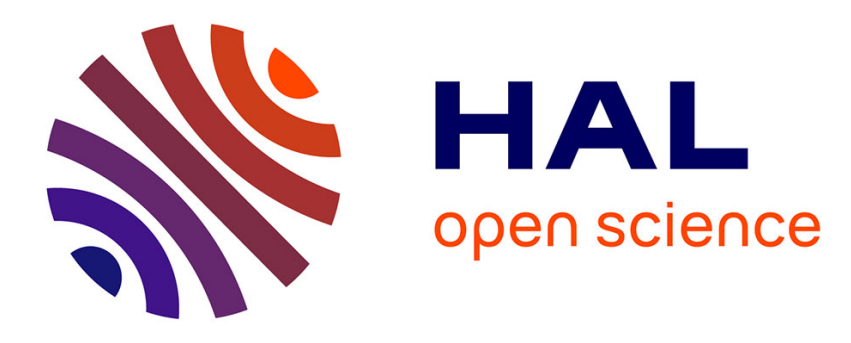

\title{
FFT based iterative schemes for composites conductors with non-overlapping fibers and Kapitza interface resistance
}

Vincent Monchiet

\section{- To cite this version: \\ Vincent Monchiet. FFT based iterative schemes for composites conductors with non-overlapping fibers and Kapitza interface resistance. International Journal of Solids and Structures, inPress. hal- 01665956}

\section{HAL Id: hal-01665956 \\ https://hal.science/hal-01665956}

Submitted on 18 Mar 2021

HAL is a multi-disciplinary open access archive for the deposit and dissemination of scientific research documents, whether they are published or not. The documents may come from teaching and research institutions in France or abroad, or from public or private research centers.
L'archive ouverte pluridisciplinaire $\mathbf{H A L}$, est destinée au dépôt et à la diffusion de documents scientifiques de niveau recherche, publiés ou non, émanant des établissements d'enseignement et de recherche français ou étrangers, des laboratoires publics ou privés. 


\title{
FFT based iterative schemes for composites conductors with non-overlapping fibers and Kapitza interface resistance
}

\author{
Vincent Monchiet ${ }^{\mathrm{a}}$, \\ ${ }^{a}$ Université Paris-Est, Laboratoire Modélisation et Simulation Multi Echelle, \\ MSME UMR8208 CNRS, 5 boulevard Descartes, 77454 Marne la Vallée Cedex, \\ France
}

\begin{abstract}
A FFT-based iterative scheme is developed for fiber-made composite conductors with a Kapitza thermal resistance between the matrix and the inclusions which involves a jump of the temperature. To reach this objective, we propose to extend the FFT methods to deal with Kapitza interface and to derive the size-dependent effective conductivity of such composites conductors. In this paper, we solve the inplane problem leading to the identification of the transverse effective conductivity. The original methods based on Fast Fourier Transform failed to solve efficiently the problems with imperfect interface which is intrinsically attributable to the use of Fourier series to describe the local fields. To reach this objective, we propose to derive an iterative scheme obtained from the weak form of the boundary value problem by considering a discretization along Fourier series and an enrichment with functions which are null outside of the inclusions. By doing so, the latter introduce explicitly the discontinuities at the interface. The stationarity point is computed by means of an iterative which uses the classic periodic Green function and a modified conductivity tensor that accounts for the interface thermal resistance. It is shown that the rate of convergence of this new iterative scheme is almost equivalent to that of the original method. The results for a composite with regularly distributed fibers are compared with Finite Element solutions. Next, the size-dependent effective conductivity is computed for random distributions of inclusions and compared with analytic estimates coming from the homogenization theory.
\end{abstract}

Key words: Composites, Conductivity, Homogenization, FFT, Imperfect interface

Email address: vincent.monchiet@univ-paris-est.fr (Vincent Monchiet). 


\section{Introduction}

Surface/interface effects have been introduced to account for the discontinuity of the local fields across the interface between two regions having distinct mechanical/physical properties. Generally, two kind of interface models are considered involving the discontinuity on the primal or the dual variable. For instance, in the case of elastic composites, the spring interface model introduces a discontinuity of the displacement which is proportional to the traction acting on the interface, see Goland and Reissner [11], Jones and Whittier [19]. Still in the context of elasticity, the interface model of Gurtin and Murdoch $[12,31]$ introduces a discontinuity of the traction but the displacement remains continuous across the interface. The relation giving the jump of the traction is given by a generalization of the Young-Laplace equation. In the context of the thermal conduction, the Kapitza model introduces a discontinuity of the temperature that is proportional to the flux acting on the interface. Initially, the Kapitza model [20] has been introduced to account for the interfacial resistance between a solid and a liquid and has been later considered at the frontier of two solids [6]. In the case of composites, the interface effects are generally attributed to the presence of a thin interphase or interfacial damage.

The determination of the effective properties of composites with imperfect interfaces has been the subject of numerous works in the framework of the homogenization theory, in the context of elasticity by Hashin [14], Sharma and Ganti [32], Chen et al. [7], Duan et al. [8], Lequang and He [15], Brisard et al. [5] and in the context of the thermal conduction by Benveniste and Miloh [2], Dunn and Taya [9], Torquato and Rintoul [35], Hashin [13], [16]. The most remarkable result is that the effective properties of the composites depends on the size of the reinforcements.

The boundary value problem of composites with imperfect interfaces can be easily solved with the Finite Element Method by doubling the DOFs at each nodes at the interfaces between the two regions to account for the displacement/temperature discontinuity. Alternatively, the problem with a discontinuity of the displacement (for elasticity problems) has been considered with the Extended Finite Element Method (XFEM) based on Level Set Functions and which has been initially introduced by Belytschko and Black [1], Moës et al. [23] and Sukumar et al. [33] for applications to crack problems. The application of the XFEM to composites with imperfect interfaces has been proposed by Yvonnet et al. [36,37], Zhu et al. [38].

In this paper we propose a FFT-based iterative scheme to compute the effective conductivity of composites with Kapitza interface resistance. The method based on FFT has been introduced by Moulinec and Suquet [27] in the context of elasticity and has received various improvements $[10,21,3,4,25,26]$. The method has been also extended to compute the transfer properties of porous solids [24,29,34,17]. Particularly, in [24], a dual formulation based on the stress field has been proposed to compute the Stokes flow problem with a sliding con- 
dition at the interface between the solid and the fluid phase. The skeleton of the porous material being rigid, the sliding condition naturally introduces a discontinuity of the velocity field. Note however that the method leads to prohibitive time calculations and computer memory saving (this has been shown in [28]) which makes the method inoperative for high dimension problems. In this paper, we propose an alternative approach which is based on the discretization of the boundary value problem in a variational procedure with Fourier series and additional local fields which explicitly introduce a discontinuity at the interface between the matrix and the inclusions. The method of resolution still uses an iterative scheme based on the Green operator and the FFT algorithm. The convergence of the new iterative scheme is studied in the case of a network of fibers with circular cross section and the solutions are compared with FE solutions. Next, we apply the method in the case of composites with randomly distributed fibers and the solutions are compared with analytic solutions coming from the homogenization theory [16]. Finally, the method is applied to a composite with a random distribution of fibers which are different in size.

\section{Homogenization problem and interface model}

Consider a periodic composite made up of a matrix with the conductivity $k_{1}$ and inclusions having the conductivity $k_{2}$. We denote by $V_{1}$ the volume of the matrix and by $V_{2}$ the volume of the inclusions. The volume $V_{2}$ can be decomposed into $\mathcal{P}$ subdomains $\Omega_{1}, \ldots, \Omega_{\mathcal{P}}$ corresponding to each isolated inclusion. In this paper, the hypothesis of non-overlapping fibers is used. The interface between these inhomogeneities and the matrix is denoted by $\Gamma_{i}$ for $i=1 \ldots \mathcal{P}$. The boundary value problem reads:

$$
\left\{\begin{array}{l}
\boldsymbol{\varepsilon}(\boldsymbol{x})=\nabla u(\boldsymbol{x}), \quad \forall \boldsymbol{x} \in V \\
\boldsymbol{\sigma}(\boldsymbol{x})=\boldsymbol{K}(\boldsymbol{x}) \cdot \boldsymbol{\varepsilon}(\boldsymbol{x}), \quad \forall \boldsymbol{x} \in V \\
\operatorname{div}(\boldsymbol{\sigma}(\boldsymbol{x}))=0, \quad \forall \boldsymbol{x} \in V \\
u-\boldsymbol{E} \cdot \boldsymbol{x} \text { periodic, } \boldsymbol{\sigma} \cdot \boldsymbol{n} \text { antiperiodic, } \quad \forall \boldsymbol{x} \in \partial V
\end{array}\right.
$$

where $\boldsymbol{E}$ is the macroscopic gradient of temperature defined by:

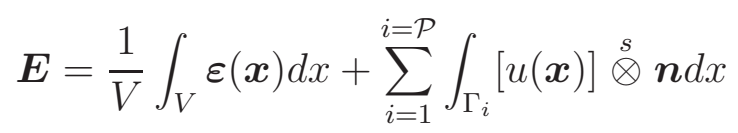


where $\boldsymbol{a} \stackrel{s}{\otimes} \boldsymbol{b}=(\boldsymbol{a} \otimes \boldsymbol{b}+\boldsymbol{b} \otimes \boldsymbol{a}) / 2$ for any vector $\boldsymbol{a}$ and $\boldsymbol{b}$.

In Eq. (1), $\boldsymbol{K}(\boldsymbol{x})$ represents the conductivity tensor of the heterogeneous medium, given by:

$$
\boldsymbol{K}(\boldsymbol{x})=I_{1}(\boldsymbol{x}) \boldsymbol{K}_{1}+I_{2}(\boldsymbol{x}) \boldsymbol{K}_{2}
$$

in which $I_{1}(\boldsymbol{x})$ and $I_{2}(\boldsymbol{x})$ are the characteristic functions describing the volume $V_{1}$ and $V_{2}$ respectively. The characteristic function $I_{2}(\boldsymbol{x})$ can be read:

$$
I_{2}(\boldsymbol{x})=\sum_{i=1}^{i=\mathcal{P}} \chi_{i}(\boldsymbol{x})
$$

where $\chi_{i}(\boldsymbol{x})$ for $i=1 \ldots \mathcal{P}$ are the characteristic functions of the volume $\Omega_{1}, \ldots, \Omega_{\mathcal{P}}$. We assume that the constituents are isotropic, $\boldsymbol{K}_{1}=k_{1} \boldsymbol{I}$, and $\boldsymbol{K}_{2}=k_{2} \boldsymbol{I}$ where $\boldsymbol{I}$ is the identity for two order tensors.

At the interface between the inclusions and the matrix, the temperature is discontinuous and the jump of the temperature is given by:

$$
[u(\boldsymbol{x})]_{\Gamma_{i}}=\left.h_{s} \boldsymbol{\sigma}(\boldsymbol{x}) \cdot \boldsymbol{n}\right|_{\Gamma_{i}}
$$

where $[u(\boldsymbol{x})]_{\Gamma_{i}}$ represents the jump of the temperature across the interface $\Gamma_{i}$, $\boldsymbol{n}$ is the normal unit vector taken on $\Gamma_{i}$ oriented from $\Omega_{i}$ to $V_{1} . h_{s}$ is thermal resistance of the interface. Particularly, $h_{s}=0$ corresponds to a perfect interface (evolving the continuity of the temperature across $\Gamma_{i}$ ).

The effective conductivity of the composite is determined by computing the macroscopic flux $\boldsymbol{\Sigma}$ from the classic average relation:

$$
\boldsymbol{\Sigma}=\frac{1}{V} \int_{V} \boldsymbol{\sigma}(\boldsymbol{x}) d x
$$

The weak form of the boundary value problem (1) with the interface condition (5) is:

$$
\Sigma: \boldsymbol{E}=\min _{u \in \mathcal{U}} W(u)
$$

with:

$$
W(u)=\frac{1}{V} \int_{V} \varepsilon(u(\boldsymbol{x})) \cdot \boldsymbol{K}(\boldsymbol{x}) \cdot \boldsymbol{\varepsilon}(u(\boldsymbol{x})) d x+\frac{1}{V} \sum_{i=1}^{i=\mathcal{P}} \int_{\Gamma_{i}} \frac{[u(\boldsymbol{x})]^{2}}{h_{s}} d x
$$

and where $\mathcal{U}$ is the set of admissible temperature fields which are periodic, continuous and differentiable in $V_{1}$ and $V_{2}$, and which satisfy to Eq. (2). 


\section{Discretization of the problem}

\subsection{Discrete problem}

The numerical homogenization method based on the FFT applied to elasticity problems can be derived from the standard variational principle and a representation of the local fields with Fourier series. This has been shown in a recent work [26]. In order to extend the approach to composites materials with imperfect interfaces we need to extend the space of discretization by adding some fields which are discontinuous across each interface $\Gamma_{i}$ for $i=1 \ldots \mathcal{P}$.

In the case of composites conductors with perfect interfaces, the temperature is expanded along Fourier series:

$$
u(\boldsymbol{x})=\sum_{n=-N}^{n=N-1} \widehat{u}_{n} \exp \left(i \boldsymbol{\xi}_{n} \cdot \boldsymbol{x}\right)
$$

where $\widehat{u}_{n}$ are the Fourier coefficients of the temperature $u(\boldsymbol{x})$ and $\boldsymbol{\xi}_{n}$ denotes the discrete wave vectors given by:

$$
\boldsymbol{\xi}_{n}=2 \pi n \boldsymbol{\zeta}, \quad n=-N . . N-1, \quad \zeta_{i}=\frac{1}{h_{i}}
$$

and $2 h_{1}, 2 h_{2}, 2 h_{3}$ are the size of the unit cell along each space directions. For simplicity, only one indice "n" is used for the discrete wave vector $\boldsymbol{\xi}_{n}$ while three would be used for $3 \mathrm{~d}$ problems.

In order to introduce the discontinuities of the temperature across the interfaces $\Gamma_{i}$, an enrichment of the space of discretization is introduced. We add to the displacement (9) a finite number of fields $U^{(i)}(\boldsymbol{x})$ which are null outside of $\Omega_{i}$ but are different of zero inside $\Omega_{i}$, including the interface $\Gamma_{i}$. The total temperature then reads:

$$
u(\boldsymbol{x})=\sum_{n=-N}^{n=N-1} \widehat{u}_{n} \exp \left(i \boldsymbol{\xi}_{n} \cdot \boldsymbol{x}\right)+\sum_{i=1}^{i=\mathcal{P}} U^{(i)}(\boldsymbol{x})
$$

Since each terms of the Fourier series are continuous, the jump of the temperature across the interface $\Gamma_{i}$ is:

$$
[u(\boldsymbol{x})]_{\Gamma_{i}}=-\left.U^{(i)}(\boldsymbol{x})\right|_{\boldsymbol{x} \in \Gamma_{i}}
$$

By derivation, we have for any $\boldsymbol{x} \in V-\Gamma_{i}$ :

$$
\boldsymbol{\varepsilon}(u(\boldsymbol{x}))=\sum_{n=-N}^{n=N-1} \widehat{\boldsymbol{\varepsilon}}_{n} \exp \left(i \boldsymbol{\xi}_{n} \cdot \boldsymbol{x}\right)+\sum_{i=1}^{i=\mathcal{P}} \boldsymbol{\varepsilon}\left(U^{(i)}(\boldsymbol{x})\right)
$$


where $\widehat{\varepsilon}_{n}$ is defined, $\forall n \neq 0$, by :

$$
\widehat{\varepsilon}_{n}=i \widehat{u}_{n} \boldsymbol{\xi}_{n}
$$

The unknowns $\widehat{\varepsilon}_{n}$ could be substituted to $\widehat{u}_{n}$ by accounting for the compatibility condition (14).

The temperature field must also comply with (2) in order to be kinematically admissible. Owing to relation (12) we deduce that:

$$
\boldsymbol{E}=\widehat{\boldsymbol{\varepsilon}}_{0}+\sum_{i=1}^{i=\mathcal{P}}\left[\frac{1}{V} \int_{\Omega_{i}} \boldsymbol{\varepsilon}\left(U^{(i)}(\boldsymbol{x})\right) d x-\frac{1}{V} \int_{\Gamma_{i}} U^{(i)}(\boldsymbol{x}) \boldsymbol{n} d x\right]
$$

It is readily observed that the last two integrals in the above equation vanish when using the gradient theorem to transform the volume integral over $\Omega_{i}$ into an integral over the surface $\Gamma_{i}$. It then remains:

$$
\boldsymbol{E}=\widehat{\varepsilon}_{0}
$$

Introducing the temperature (11) in (8), we obtain:

$$
\begin{aligned}
& W(u)=\sum_{n=-N}^{n=N-1} \sum_{m=-N}^{m=N-1} \overline{\widehat{\varepsilon}_{n}} \cdot \widehat{\boldsymbol{K}}\left(\boldsymbol{\xi}_{n}-\boldsymbol{\xi}_{m}\right) \cdot \widehat{\boldsymbol{\varepsilon}}_{m}+2 \sum_{n=-N}^{n=N-1} \sum_{i=1}^{i=\mathcal{P}} \overline{\widehat{\boldsymbol{\varepsilon}}_{n}} \cdot \boldsymbol{K}_{2} \cdot \widehat{\boldsymbol{D}}_{n}^{(i)} \\
& +\sum_{i=1}^{i=\mathcal{P}} \Psi^{(i)}\left(U^{(i)}(\boldsymbol{x}), U^{(i)}(\boldsymbol{x})\right)
\end{aligned}
$$

where $\overline{\widehat{\varepsilon}_{n}}$ represents the conjugate of $\widehat{\boldsymbol{\varepsilon}}$ and $\widehat{\boldsymbol{K}}(\boldsymbol{\xi})$ is the Fourier transform of the conductivity tensor $\boldsymbol{K}(\boldsymbol{x})$. The first term in the above equation, involving the double sum over $n$ and $m$, is derived considering the Parseval theorem. Also the second term in Eq. (17) is obtained with the Parseval theorem and the following definition:

$$
\widehat{\boldsymbol{D}}_{n}^{(i)}=\frac{1}{V} \int_{V} \boldsymbol{\varepsilon}\left(U^{(i)}(\boldsymbol{x})\right) \exp \left(-i \boldsymbol{\xi}_{n} \cdot \boldsymbol{x}\right) d x
$$

In the last term in Eq. (17), the function $\Psi^{(i)}$ is defined by:

$$
\Psi^{(i)}(u(\boldsymbol{x}), v(\boldsymbol{x}))=\frac{1}{V} \int_{\Omega_{i}} \boldsymbol{\varepsilon}(u(\boldsymbol{x})) \cdot \boldsymbol{K}_{2} \cdot \boldsymbol{\varepsilon}(v(\boldsymbol{x})) d x+\frac{1}{V} \int_{\Gamma_{i}} \frac{u(\boldsymbol{x}) v(\boldsymbol{x})}{h_{s}} d x
$$

In each subdomain $\Omega_{i}$ the displacement is represented along a basis of interpolation functions: 


$$
U^{(i)}(\boldsymbol{x})=\sum_{j=1}^{j=\mathcal{Q}} \lambda_{j}^{(i)} w_{j}^{(i)}(\boldsymbol{x}) \chi^{(i)}(\boldsymbol{x})
$$

where $\lambda_{j}^{(i)}$ are the unknown coefficients, $w_{j}^{(i)}(\boldsymbol{x})$ are the interpolation functions and $\chi^{(i)}(\boldsymbol{x})$ are the characteristic functions of the domain $\Omega_{i}$ for $i=1 . . \mathcal{P}$. Introducing expression (20) in Eq. (18), it leads to:

$$
\widehat{\boldsymbol{D}}_{n}^{(i)}=\sum_{j=1}^{j=\mathcal{Q}} \lambda_{j}^{(i)} \boldsymbol{\pi}_{j}^{(i)}\left(\boldsymbol{\xi}_{n}\right)
$$

where the vectors $\boldsymbol{\pi}_{j}^{(i)}(\boldsymbol{\xi})$ for $j=1 \ldots \mathcal{Q}$ are given by:

$$
\boldsymbol{\pi}_{j}^{(i)}\left(\boldsymbol{\xi}_{n}\right)=\frac{1}{V} \int_{\Omega_{i}} \varepsilon\left(w_{j}^{(i)}(\boldsymbol{x})\right) \exp \left(-i \boldsymbol{\xi}_{n} \cdot \boldsymbol{x}\right) d x
$$

Introducing (20) in Eq. (19), it leads to:

$$
\Psi^{(i)}\left(U^{(i)}(\boldsymbol{x}), U^{(i)}(\boldsymbol{x})\right)=\sum_{j=1}^{j=\mathcal{Q}} \sum_{k=1}^{k=\mathcal{Q}} \lambda_{j}^{(i)} \lambda_{k}^{(i)} \mathcal{A}_{j k}^{(i)}
$$

with:

$$
\mathcal{A}_{j k}^{(i)}=\Psi^{(i)}\left(\boldsymbol{w}_{j}^{(i)}(\boldsymbol{x}), \boldsymbol{w}_{k}^{(i)}(\boldsymbol{x})\right)
$$

It follows that $W(u)$ reads:

$$
\begin{aligned}
W(u)= & \sum_{n=-N}^{n=N-1} \sum_{m=-N}^{m=N-1} \overline{\widehat{\varepsilon}_{n}} \cdot \widehat{\boldsymbol{K}}\left(\boldsymbol{\xi}_{n}-\boldsymbol{\xi}_{m}\right) \cdot \widehat{\boldsymbol{\varepsilon}}_{m} \\
& +2 \sum_{n=-N}^{n=N-1} \sum_{i=1}^{i=\mathcal{P}} \sum_{j=1}^{j=\mathcal{Q}} \lambda_{j}^{(i)} \overline{\widehat{\varepsilon}_{n}} \cdot \boldsymbol{K}_{2} \cdot \boldsymbol{\pi}_{j}^{(i)}\left(\boldsymbol{\xi}_{n}\right) \\
& +\sum_{i=1}^{i=\mathcal{P}} \sum_{j=1}^{j=\mathcal{Q}} \sum_{k=1}^{k=\mathcal{Q}} \lambda_{j}^{(i)} \mathcal{A}_{j k}^{(i)} \lambda_{k}^{(i)}
\end{aligned}
$$

\subsection{The minimization}

The stationarity point of $W(u)$ given by Eq. (25) is computed by minimizing with respect to $\widehat{\varepsilon}_{n}$ for $n=-N . . N-1$ and $\lambda_{j}^{(i)}$ for $j=1 . . \mathcal{Q}$ and $i=1 . . \mathcal{P}$. Note 
that we have substituted to the true unknowns $\widehat{u}_{n}$ the Fourier coefficients $\widehat{\varepsilon}_{n}$ of the temperature gradient, so, when doing the minimization, the comparability equation (14) must be accounted for.

The compatibility condition can be also written by considering two projectors $\boldsymbol{P}_{n}$ and $\boldsymbol{Q}_{n}$ already introduced by Milton [22]. The use of these projectors is essential for the minimization procedure and also for the derivation of the iterative scheme which leads to the computation of the solution at the stationarity point.

These projectors are defined by:

$$
\boldsymbol{P}_{n}=\frac{1}{\left\|\boldsymbol{\xi}_{n}\right\|^{2}} \boldsymbol{\xi}_{n} \otimes \boldsymbol{\xi}_{n}, \quad \boldsymbol{Q}_{n}=\boldsymbol{I}-\boldsymbol{P}_{n}
$$

for any $n \neq 0$ and $\boldsymbol{P}_{n}=\boldsymbol{Q}_{n}=0$ when $n=0$.

The compatibility condition (14) for the Fourier coefficients of the gradient of temperature $\widehat{\varepsilon}_{n}$ is:

$$
Q_{n} \cdot \widehat{\varepsilon}_{n}=0
$$

or alternatively:

$$
\boldsymbol{P}_{n} . \widehat{\varepsilon}_{n}=\widehat{\varepsilon}_{n}
$$

for any $n \neq 0$.

Note that these two projectors can be also used to express the equilibrium of the flux in the Fourier space. Indeed, by denoting $\widehat{\boldsymbol{\sigma}}_{n}$ the Fourier coefficients of a divergence free field $\boldsymbol{\sigma}$, they satisfy to:

$$
\boldsymbol{P}_{n} . \widehat{\boldsymbol{\sigma}}_{n}=0
$$

or equivalently:

$$
\boldsymbol{Q}_{n} \cdot \widehat{\boldsymbol{\sigma}}_{n}=\widehat{\boldsymbol{\sigma}}_{n}
$$

The minimum of $(25)$ with respect to $\widehat{\varepsilon}_{n}$ is:

$$
\boldsymbol{P}_{n} \cdot\left[\sum_{m=-N}^{m=N-1} \widehat{\boldsymbol{K}}\left(\boldsymbol{\xi}_{n}-\boldsymbol{\xi}_{m}\right) \cdot \widehat{\boldsymbol{\varepsilon}}_{m}+\sum_{i=1}^{i=\mathcal{P}} \sum_{j=1}^{j=\mathcal{Q}} \lambda_{j}^{(i)} k_{2} \boldsymbol{\pi}_{j}^{(i)}\left(\boldsymbol{\xi}_{n}\right)\right]=0
$$

where $\boldsymbol{K}_{2}$ has been replaced by $k_{2} \boldsymbol{I}$ (we assume that the phases are isotropic). The minimum with respect to $\lambda_{j}^{(i)}$ is: 


$$
\begin{cases}\sum_{n=-N}^{n=N-1} k_{2} \overline{\widehat{\varepsilon}_{n}} \cdot \boldsymbol{\pi}_{j}^{(1)}\left(\boldsymbol{\xi}_{n}\right)+\sum_{k=1}^{k=\mathcal{Q}} \mathcal{A}_{j k}^{(1)} \lambda_{k}^{(1)}=0 & \text { for } j=1 . . \mathcal{Q}, \\ \sum_{n=-N}^{n=N-1} k_{2} \overline{\widehat{\varepsilon}_{n}} \cdot \boldsymbol{\pi}_{j}^{(2)}\left(\boldsymbol{\xi}_{n}\right)+\sum_{k=1}^{k=\mathcal{Q}} \mathcal{A}_{j k}^{(2)} \lambda_{k}^{(2)}=0 & \text { for } j=1 . . \mathcal{Q}, \\ \vdots & \\ \sum_{n=-N}^{n=N-1} k_{2}{\overline{\widehat{\varepsilon}_{n}}}_{\boldsymbol{\pi}_{j}} \boldsymbol{\pi}_{j}^{(\mathcal{P})}\left(\boldsymbol{\xi}_{n}\right)+\sum_{k=1}^{k=\mathcal{Q}} \mathcal{A}_{j k}^{(\mathcal{P})} \lambda_{k}^{(\mathcal{P})}=0 & \text { for } j=1 . . \mathcal{Q}\end{cases}
$$

Introducing the inverse of $\mathcal{A}_{j k}^{(i)}$ by $\mathcal{R}_{j k}^{(i)}$, we can eliminate the unknowns $\lambda_{j}^{(i)}$ for $j=1 \ldots \mathcal{Q}$ and for $i=1 . . \mathcal{P}$ from Eqs (31) and (32). The values at the minimum for $\widehat{\varepsilon}_{n}$ with $n=-N . . N-1$ satisfy to:

$$
\boldsymbol{P}_{n} \cdot \sum_{m=-N}^{m=N-1} \boldsymbol{L}_{n, m} \cdot \widehat{\varepsilon}_{m}=0
$$

for $n=-N . . N-1$ excepted for $n=0$ for which $\widehat{\varepsilon}_{0}=\boldsymbol{E}$ and where the fourth order tensor $\boldsymbol{L}_{n, m}$ is given by:

$$
\boldsymbol{L}_{n, m}=\widehat{\boldsymbol{K}}\left(\boldsymbol{\xi}_{n}-\boldsymbol{\xi}_{m}\right)-k_{2}^{2} \sum_{i=1}^{i=\mathcal{P}} \sum_{j=1}^{j=\mathcal{Q}} \sum_{k=1}^{k=\mathcal{Q}} \mathcal{R}_{j k}^{(i)} \boldsymbol{\pi}_{j}^{(i)}\left(\boldsymbol{\xi}_{n}\right) \otimes \overline{\boldsymbol{\pi}}_{k}^{(i)}\left(\boldsymbol{\xi}_{m}\right)
$$

Introducing:

$$
\widehat{\boldsymbol{\sigma}}_{n}=\sum_{m=-N}^{m=N-1} \boldsymbol{L}_{n, m} \cdot \widehat{\boldsymbol{\varepsilon}}_{m}
$$

we deduce that the value of $W(u(\boldsymbol{x}))$ at the stationarity point is:

$$
W(u(\boldsymbol{x}))=\sum_{n=-N}^{n=N-1} \widehat{\boldsymbol{\sigma}}_{n} \cdot \overline{\widehat{\varepsilon}_{n}}
$$

Moreover, $\widehat{\boldsymbol{\sigma}}_{n}$ complies with Eq. (29) and $\widehat{\boldsymbol{\varepsilon}}_{n}$ complies with Eq. (27), consequently, $\widehat{\boldsymbol{\sigma}}_{n}$ and $\widehat{\boldsymbol{\varepsilon}}_{n}$ are orthogonal $\forall n \neq 0$. In the sum for $n=-N . . N-1$ between $\widehat{\boldsymbol{\sigma}}_{n}$ and $\widehat{\boldsymbol{\varepsilon}}_{n}$, it then only remains the term corresponding to $n=0$ :

$$
W(u(\boldsymbol{x}))=\widehat{\boldsymbol{\sigma}}_{0} \cdot \overline{\widehat{\varepsilon}_{0}}=\widehat{\boldsymbol{\sigma}}_{0} \cdot \boldsymbol{E}
$$

$\widehat{\boldsymbol{\sigma}}_{0}$ represents the macroscopic flux which satisfies to $\widehat{\boldsymbol{\sigma}}_{0}=\boldsymbol{K}^{\text {hom }} \cdot \boldsymbol{E}$ where $\boldsymbol{K}^{\text {hom }}$ is the homogenized or effective conductivity of the composite. The ef- 
fective conductivity is then determined by considering $\widehat{\boldsymbol{\sigma}}_{0}$ at convergence of the iterative scheme.

\subsection{Resolution with an iterative scheme}

The solution of the Eq. (33) can be computed by means of the following iterative scheme:

$$
\widehat{\boldsymbol{\varepsilon}}_{n}^{r+1}=\widehat{\boldsymbol{\varepsilon}}_{n}^{r}-\widehat{\boldsymbol{\Gamma}}_{n}^{0} \cdot\left[\sum_{m=-N}^{m=N-1} \boldsymbol{L}_{n, m} \widehat{\boldsymbol{\varepsilon}}_{m}^{r}\right]
$$

where $\widehat{\boldsymbol{\varepsilon}}_{n}^{r}$ is the value of $\widehat{\boldsymbol{\varepsilon}}_{n}$ computed at iteration " $r$ ". The iterative scheme is initialized with the macroscopic gradient of temperature $\boldsymbol{E}$ :

$$
\widehat{\boldsymbol{\varepsilon}}_{n}^{1}= \begin{cases}0 & \forall n \neq 0 \\ \boldsymbol{E} & \text { for } n=0\end{cases}
$$

In Eq. (38), $\widehat{\boldsymbol{\Gamma}}_{n}^{0}$ are the Fourier coefficients of the Green tensor associated to a reference medium of conductivity $k_{0}$. Its components are:

$$
\widehat{\boldsymbol{\Gamma}}_{n}^{0}=\frac{1}{k_{0}} \boldsymbol{P}_{n}
$$

where $k_{0}$ can be interpreted as a preconditioner that is adjusted in order to obtain the best convergence of the iterative scheme. Its value is discussed in section 4.2. Comparatively to the original method of Moulinec and Suquet [27], the discontinuity of the temperature is introduced with the extra term $" \mathcal{R}_{j k}^{(i)} \boldsymbol{\pi}_{j}^{(i)}\left(\boldsymbol{\xi}_{n}\right) \otimes \boldsymbol{\pi}_{k}^{(i)}\left(\boldsymbol{\xi}_{m}\right)$ " in the definition of $\boldsymbol{L}_{n, m}$ given by Eq. (34). In Eq. (38), the discrete convolution product between $\widehat{\boldsymbol{K}}\left(\boldsymbol{\xi}_{n}-\boldsymbol{\xi}_{m}\right)$ and $\widehat{\boldsymbol{\varepsilon}}_{m}^{r}$ is effected with the FFT algorithm. In the other hand, the product " $\mathcal{R}_{j k}^{(i)} \boldsymbol{\pi}_{j}^{(i)}\left(\boldsymbol{\xi}_{n}\right) \otimes \boldsymbol{\pi}_{k}^{(i)}\left(\boldsymbol{\xi}_{m}\right)$ " with $\widehat{\boldsymbol{\varepsilon}}_{m}^{r}$ is local in the Fourier space. The sum over the indice $m$ between $\boldsymbol{\pi}_{k}^{(i)}\left(\boldsymbol{\xi}_{m}\right)$ and $\widehat{\boldsymbol{\varepsilon}}_{m}^{r}$ just consists of a Hadamard product between two tables and by summing all the components.

As in the original method, the implementation of the problem only requires the storage of tables of dimension $N_{t}=(2 N)^{2}$ in 2 d case and $N_{t}=(2 N)^{3}$ for 3 d one. Note that $N_{t}$ represents the total number of Fourier components of the temperature (see Eq (9)) and then the degree of Freedom (DOFs) for the problem of composite with perfect interfaces. In the case of imperfect interface, the DOFs is $N_{t}$ plus the total number of interpolation functions that is $\mathcal{P} \times \mathcal{Q}$. In the original FFT method applied to composite conductors with perfect 
interfaces, the implementation requires the storage of tables of dimension $N_{t}$ that is the components of the gradient of temperature $\widehat{\boldsymbol{\varepsilon}}_{n}^{r}$ (that is 2 tables), the flux $\boldsymbol{\sigma}_{n}^{r}$ (that is 2), the local conductivity $k\left(\boldsymbol{x}_{n}\right)$ (that is 1 for isotropic constituents) and finally the components of the Green tensor (that is 6). In the version of the method which uses the shape function, the storage of $\chi(\boldsymbol{\xi})$ is used instead of $k\left(\boldsymbol{x}_{n}\right)$. So the total number of tables of dimension $N_{t}$ is $2+2+1+6=11$. The algorithm developed here for imperfect interfaces requires the storage of $\mathcal{P} \times \mathcal{Q}$ additional matrices: $\boldsymbol{\pi}_{j}^{(i)}\left(\boldsymbol{\xi}_{n}\right)$ for $i=1 . . \mathcal{P}$ and $j=1 . . \mathcal{Q}$. Obviously, the method is numerically interesting only if the dimension of the space of discretization of the functions $U^{(i)}(\boldsymbol{x})$, defined by $\mathcal{Q}$, is not too large. As shown in the next of the paper when all the inclusions are identical in shape and size the total number of tables reduce to $\mathcal{Q}$ instead of $\mathcal{P} \times \mathcal{Q}$.

\section{Application to a composite with regularly distributed fibers}

We apply the method to the case of a composite reinforced by long fibers aligned in the direction $O x_{3}$. The fibers are regularly distributed in the directions $O x_{1}$ and $O x_{2}$. The unit cell contains one fiber as illustrated in Fig. 1. The case of a population of $\mathcal{P}$ inclusions in the unit cell is studied in the next section.

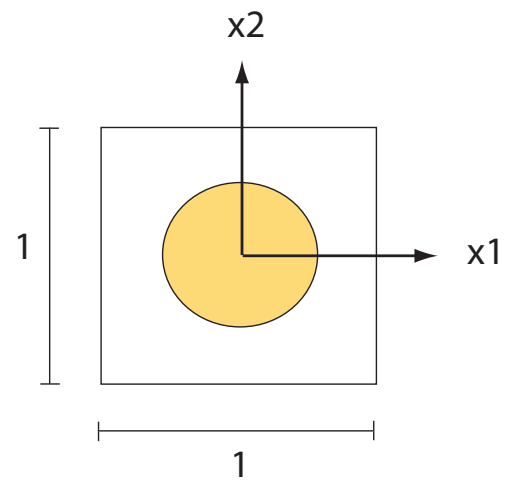

Fig. 1. Unit cell of a composite with regularly distributed fibers.

\subsection{The choice of the interpolation functions}

In the case of a composite with aligned cylindrical fibers with the radius $R$, the Fourier transform of the characteristic function of the inclusion $\chi(\boldsymbol{x})$ has a close-form expression in the Fourier space. This expression can be found in the book of Nemat-Nasser [30] and has been used to formulate estimations of the effective elastic properties of composites. This exact expression, called shape function in [30], has been also used in [3] to improve the solutions of 
standard FFT-based iterative schemes. Later, in [26], it has been proved that the use of the shape functions in the FFT-based iterative schemes provides rigorous bounds of the effective elastic properties of the composites. Note that the approach developed in the present paper is also based on a variational procedure which garante the upper bound character of the numerical solution. The derivation of a lower bound is out of reach of this work.

Considering the particular case of a cylinder of radius $R$, the associated shape function is:

$$
\widehat{\chi}(\boldsymbol{\xi})= \begin{cases}\frac{2 c}{R\|\boldsymbol{\xi}\|} J_{1}(R\|\boldsymbol{\xi}\|) & \forall \boldsymbol{\xi} \neq 0 \\ c & \boldsymbol{\xi}=0\end{cases}
$$

where $c=\pi R^{2} / V$ and $V=4 h_{1} h_{2}$ where it is recalled that $2 h_{1}$ and $2 h_{2}$ denote the dimensions of the cell along the directions $O x_{1}$ and $O x_{2}$ respectively that is equal to 1 in this example. In the above equation, $\|\boldsymbol{\xi}\|$ is the norm of the wave vector $\boldsymbol{\xi}$ and $J_{n}$ for $n=0,1,2, \ldots$ are the bessel functions of the first kind.

Since the unit cell contains only one inclusion, the superscript "(i)" can be omitted in this section. In the inclusion (the domain $\Omega$ ), we use orthogonal polynomials for the interpolation functions. Assuming that the inclusion is centered at $\boldsymbol{x}=0$, the first nine interpolation functions are:

$$
\begin{aligned}
& w_{1}(\boldsymbol{x})=x_{1} \\
& w_{2}(\boldsymbol{x})=x_{2} \\
& w_{3}(\boldsymbol{x})=x_{1}^{2}+x_{2}^{2}-R^{2} \\
& w_{4}(\boldsymbol{x})=x_{1} x_{2} \\
& w_{5}(\boldsymbol{x})=x_{2}^{2}-x_{1}^{2} \\
& w_{6}(\boldsymbol{x})=x_{1}\left(x_{1}^{2}-3 x_{2}^{2}\right) \\
& w_{7}(\boldsymbol{x})=x_{2}\left(x_{2}^{2}-3 x_{1}^{2}\right) \\
& w_{8}(\boldsymbol{x})=x_{1}\left(x_{1}^{2}+x_{2}^{2}-R^{2}\right) \\
& w_{9}(\boldsymbol{x})=x_{2}\left(x_{1}^{2}+x_{2}^{2}-R^{2}\right)
\end{aligned}
$$

Interestingly, it can be observed that: 


$$
\frac{1}{V} \int_{\Omega} \underbrace{x_{i} \ldots x_{j}}_{\mathrm{n} \text { times }} \exp (-i \boldsymbol{\xi} \cdot \boldsymbol{x}) d x=(-i)^{n} \frac{\partial}{\partial \xi_{i}} \ldots \frac{\partial}{\partial \xi_{j}} \widehat{\chi}(\boldsymbol{\xi})
$$

It follows that all the vectors $\boldsymbol{\pi}_{j}(\boldsymbol{\xi})$ defined by Eq. (22) can be expressed in term of the derivatives of the shape function $\widehat{\chi}(\boldsymbol{\xi})$ :

$$
\begin{aligned}
& \boldsymbol{\pi}_{1}(\boldsymbol{\xi})=\widehat{\chi}(\boldsymbol{\xi}) \boldsymbol{e}_{1} \\
& \boldsymbol{\pi}_{2}(\boldsymbol{\xi})=\widehat{\chi}(\boldsymbol{\xi}) \boldsymbol{e}_{2} \\
& \boldsymbol{\pi}_{3}(\boldsymbol{\xi})=-2 i \widehat{\chi}_{, 1}(\boldsymbol{\xi}) \boldsymbol{e}_{1}-2 i \widehat{\chi}_{, 2}(\boldsymbol{\xi}) \boldsymbol{e}_{2} \\
& \boldsymbol{\pi}_{4}(\boldsymbol{\xi})=-i \widehat{\chi}_{, 2}(\boldsymbol{\xi}) \boldsymbol{e}_{1}-i \widehat{\chi}, 1(\boldsymbol{\xi}) \boldsymbol{e}_{2} \\
& \boldsymbol{\pi}_{5}(\boldsymbol{\xi})=-2 i \widehat{\chi}_{, 2}(\boldsymbol{\xi}) \boldsymbol{e}_{2} \\
& \boldsymbol{\pi}_{6}(\boldsymbol{\xi})=3\left[\widehat{\chi}_{, 22}(\boldsymbol{\xi})-\widehat{\chi}_{, 11}(\boldsymbol{\xi})\right] \boldsymbol{e}_{1}+6 \widehat{\chi}_{, 12}(\boldsymbol{\xi}) \boldsymbol{e}_{2} \\
& \boldsymbol{\pi}_{7}(\boldsymbol{\xi})=6 \widehat{\chi}_{, 12}(\boldsymbol{\xi}) \boldsymbol{e}_{1}+3\left[\widehat{\chi}_{, 11}(\boldsymbol{\xi})-\widehat{\chi}_{, 22}(\boldsymbol{\xi})\right] \boldsymbol{e}_{2} \\
& \boldsymbol{\pi}_{8}(\boldsymbol{\xi})=-\left[3 \widehat{\chi}_{, 11}(\boldsymbol{\xi})+\widehat{\chi}_{, 22}(\boldsymbol{\xi})+R^{2} \chi(\boldsymbol{\xi})\right] \boldsymbol{e}_{1}-2 \widehat{\chi}_{, 12}(\boldsymbol{\xi}) \boldsymbol{e}_{2} \\
& \boldsymbol{\pi}_{9}(\boldsymbol{\xi})=-2 \widehat{\chi}_{, 12}(\boldsymbol{\xi}) \boldsymbol{e}_{1}-\left[\widehat{\chi}, 11(\boldsymbol{\xi})+3 \widehat{\chi}_{, 22}(\boldsymbol{\xi})+R^{2} \chi(\boldsymbol{\xi})\right] \boldsymbol{e}_{2}
\end{aligned}
$$

where $\hat{\chi}_{, i}$ represents the derivative of $\hat{\chi}(\boldsymbol{\xi})$ with respect to $\xi_{i}$. These relations give the vectors $\boldsymbol{\pi}_{j}(\boldsymbol{\xi})$ for any $\boldsymbol{\xi} \neq 0$. For $\boldsymbol{\xi}=0$, the values of $\boldsymbol{\pi}_{1}(\boldsymbol{\xi})$ and $\boldsymbol{\pi}_{2}(\boldsymbol{\xi})$ are equal to $c \boldsymbol{e}_{1}$ and $c \boldsymbol{e}_{2}$ where $\boldsymbol{e}_{1}$ and $\boldsymbol{e}_{2}$ are the unit vectors taken along directions $O x_{1}$ and $O x_{2}$ respectively. Vectors $\boldsymbol{\pi}_{j}(\boldsymbol{\xi})$ for $j=3,4, \ldots$ are null for $\boldsymbol{\xi}=0$.

The matrix $\mathcal{R}_{i j}$ is diagonal and its components are:

$$
\begin{aligned}
& \mathcal{R}_{11}=\mathcal{R}_{22}=\frac{1}{c k_{2}} \frac{\alpha}{1+\alpha} \\
& \mathcal{R}_{33}=\frac{1}{2 c k_{2} R^{2}}, \quad \mathcal{R}_{44}=\frac{4}{c k_{2} R^{2}} \frac{\alpha}{1+2 \alpha} \\
& \mathcal{R}_{55}=\frac{1}{c k_{2} R^{2}} \frac{\alpha}{1+2 \alpha}, \quad \mathcal{R}_{66}=\mathcal{R}_{77}=\frac{1}{c k_{2} R^{4}} \frac{\alpha}{1+3 \alpha} \\
& \mathcal{R}_{88}=\mathcal{R}_{99}=\frac{3}{2 c k_{2} R^{4}}
\end{aligned}
$$

where we have introduced the non dimensional parameter: 


$$
\alpha=\frac{k_{2} h_{s}}{R}
$$

that is null for a perfect interface.

Moreover, since the matrix $\mathcal{R}_{i j}$ is diagonale the double sum over $j$ and $k$ is replaced by a simple sum over $j$ :

$$
\boldsymbol{L}_{n, m}=\widehat{\boldsymbol{K}}\left(\boldsymbol{\xi}_{n}-\boldsymbol{\xi}_{m}\right)-k_{2}^{2} \sum_{j=1}^{j=\mathcal{Q}} \mathcal{R}_{j j} \boldsymbol{\pi}_{j}\left(\boldsymbol{\xi}_{n}\right) \otimes \overline{\boldsymbol{\pi}_{j}}\left(\boldsymbol{\xi}_{m}\right)
$$

For instance, considering only $w_{1}(\boldsymbol{x})$ and $w_{2}(\boldsymbol{x})$, in the computation of $\boldsymbol{L}_{n, m}$, we obtain (after some elementary simplifications):

$$
\boldsymbol{L}_{n, m}=\widehat{\boldsymbol{K}}\left(\boldsymbol{\xi}_{n}-\boldsymbol{\xi}_{m}\right)-\frac{k_{2}}{c} \frac{\alpha}{1+\alpha} \widehat{\chi}\left(\boldsymbol{\xi}_{n}\right) \widehat{\chi}\left(\boldsymbol{\xi}_{m}\right) \boldsymbol{I}
$$

It must be observed that for the cylindrical shape some interpolation functions do not generate a discontinuity of the temperature across the interface. These functions are $w_{3}(\boldsymbol{x}), w_{8}(\boldsymbol{x})$ and $w_{9}(\boldsymbol{x})$ which are proportional to $x_{1}^{2}+x_{2}^{2}-R^{2}$ that is null at the interface. A consequence is that the corresponding components of the matrix $\mathcal{R}_{i j}$ are independent of $\alpha$. The use of these interpolation functions for the description of the interface discontinuity of the temperature is not pertinent and can be eliminated. However, for other inclusion shapes (rectangular inclusion for instance) these fields must be kept.

The geometry of the unit cell and the applied loading indicates that the gradient of temperature into the inclusion must be an even function with respect to the components of the position vector $\boldsymbol{x}$. This suggests that the function $w_{4}(\boldsymbol{x})$ and $w_{5}(\boldsymbol{x})$ can be also omitted in the computation of the linear operator $\boldsymbol{L}_{n, m}$. Finally, only $w_{1}(\boldsymbol{x}), w_{2}(\boldsymbol{x}), w_{6}(\boldsymbol{x})$ and $w_{7}(\boldsymbol{x})$ are considered in this application.

Higher order polynomials are provided in appendix A and are used later for comparison with FE solutions. The solution which uses the polynomials of first degree, i.e. $w_{1}(\boldsymbol{x})$ and $w_{2}(\boldsymbol{x})$ (that leading to the linear operator (48)) is denoted FFT-P1. The solution based with polynomials of third degrees, $w_{1}(\boldsymbol{x})$, $w_{2}(\boldsymbol{x}), w_{6}(\boldsymbol{x})$ and $w_{7}(\boldsymbol{x})$ is denoted FFT-P3. Since the functions of even degree are not considered, there is no FFT-P2 solution. Higher order polynomials are also investigated in order to check the accuracy of the FFT-P3 solution. Polynomials of fourth degree are not considered still due to the symmetry of the problem. However, the FFT-P5 solution is considered, using fifth degree polynomials given in appendix A. 


\subsection{Analysis of convergence}

A crucial point in the application of the FFT-based iterative schemes concerns their analysis of convergence. The rate of convergence of the iterative schemes depends on the choice of the reference material $k_{0}$ which can be interpreted as a preconditioner associated with the linear system (33). The convergence of the standard FFT-based iterative scheme for elastic composites and composite conductors has been studied by Moulinec and Suquet [27], Michel et al. [21], Milton [22] by minimizing the spectral radius of the linear operator involved in the recurrence relation. In the case of a two-phases composites conductors, the convergence is guaranteed if:

$$
k_{0}>\frac{1}{2} \max \left(k_{1}, k_{2}\right)
$$

The minimization of the spectral radius leads to the following optimal conductivity of the reference material:

$$
k_{0}=\frac{k_{1}+k_{2}}{2}
$$

The case of composites conductors with imperfect interfaces introduces a third parameter, the interface resistance $h_{s}$. The method introduced in $[27,21]$ consists to bound the spectral radius of the linear operator involved by the recurrence relation but is not applicable here due to the enrichment with the interpolation functions. 

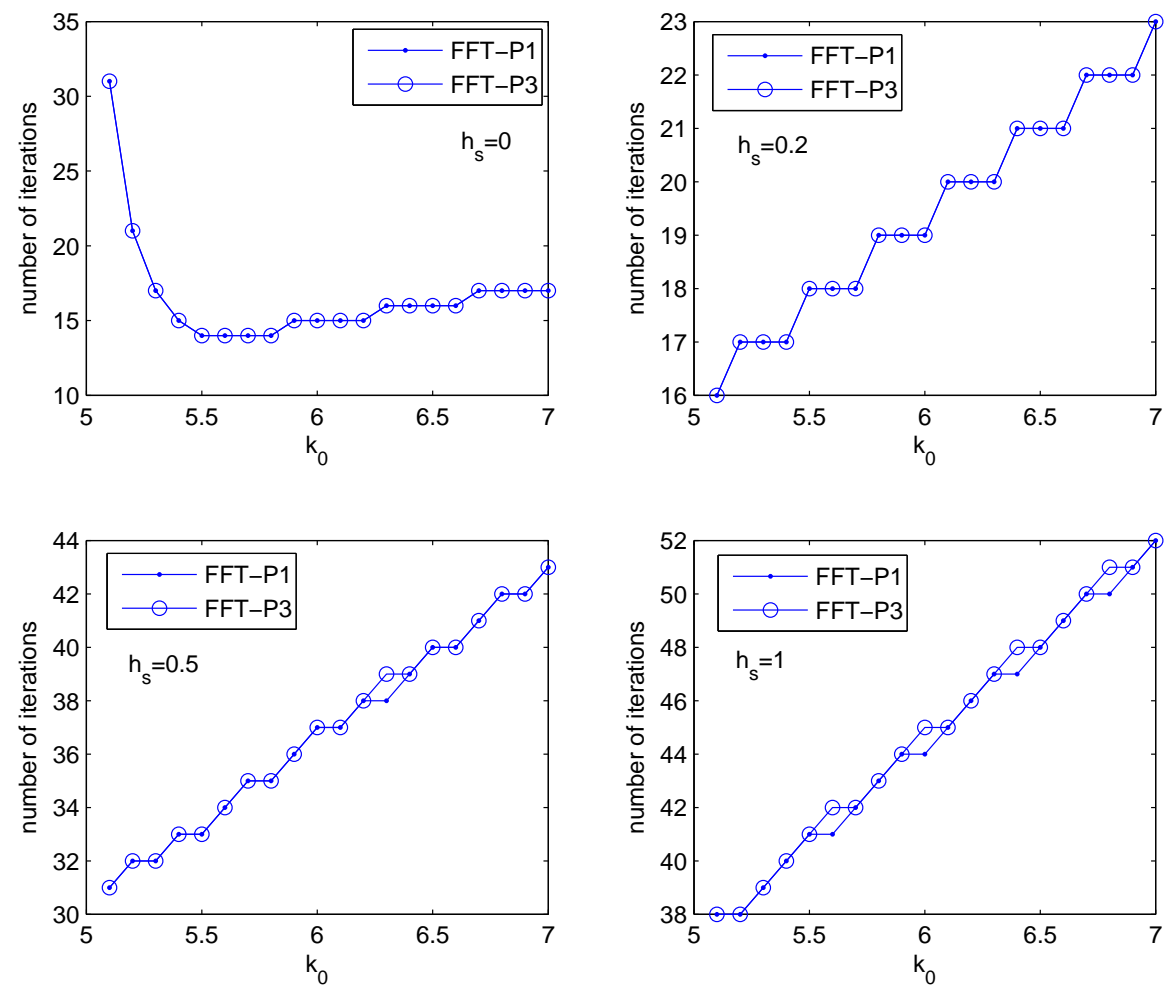

Fig. 2. Number of iteration at convergence of the iterative scheme as function of $k_{0}$ for $k_{1}=1, k_{2}=10$ and $R=0.5$.

Due to the incapacity to determine the condition giving the convergence from a theoretically point of view, we study the rate of convergence of the iterative scheme numerically. We consider a fiber with the radius $R=0.25$ (the size of the unit cell in each space direction is taken as 1). The conductivity of the matrix is $k_{1}=1$, the conductivity of the inclusion is $k_{2}=10$. In the case of a perfect interface, the convergence is guaranteed if $k_{0}>5$ (owing to relation (49)) and the optimal value corresponds to $k_{0}=5.5$ (owing to relation (50)). On Fig. 2, we represent the number of iterations at convergence of the iterative scheme as function of $k_{0}$ for $h_{s}=0, h_{s}=0.2, h_{s}=0.5, h_{s}=1$. The calculations are performed with 128 wave vectors along each space directions. For $h_{s}=0$ (case of a perfect interface), the curve has a minimum at $k_{0}=5.5$ that corresponds to the theoretical value given by Eq. (50). For other values of $h_{s}$, there is no minimum, the number of iterations monotonically increases with $k_{0}$ and diverges when $k_{0}<5$. From a practical point of view, we consider the value of the conductivity of the reference medium given by Eq. (50) whatever the value of $h_{s}$ and for all the applications proposed in the next of the paper. Also, it must be noted that the rate of convergence is the same for FFT-P1 and FFT-P3. 
On Fig. 3 is represented the variations of the number of iterations at convergence as function of the phase contrast. The conductivity of the matrix is $k_{1}=1$ and we increase the conductivity of the inclusion from 1 to 100 . Three values of the interface coefficient are considered: $h_{s}=0, h_{s}=0.5$ and $h_{s}=1$. The results show that the number of iterations has an affine relation with the contrast but the slope of each curves remains independent of the interface resistance $h_{s}$.

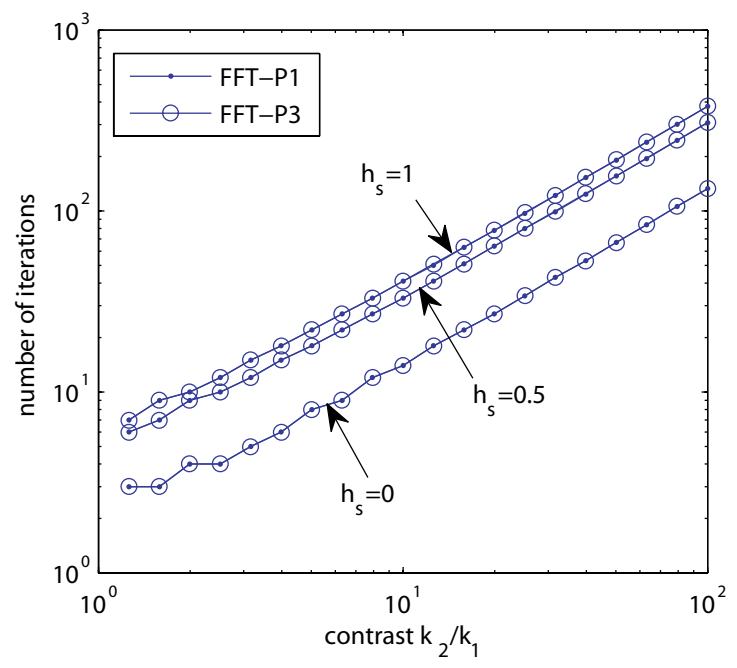

Fig. 3. Number of iterations at convergence as function $k_{2}$ for $k_{1}=1, R=0.25$ and $h_{s}=0,0.5,1$.

The variation of the number of iterations at convergence as function of $h_{s}$ is now provided on Fig. 4. The conductivity of the matrix and the inclusions are $k_{1}=1$ and $k_{2}=10$ respectively. The radius of the fiber is $R=0.25$. The number of iterations monotonically increases with $h_{s}$ and saturates at higher values of this parameter. This result shows that the rate of convergence is not strongly affected by $h_{s}$. At finite values of the contrast between the phase, the iterative scheme converges whatever the value of $h_{s}$. Obviously, by increasing the contrast, the rate of convergence decreases, that is the main fault of the FFT-based iterative scheme formulated with the strain. The particular case of a rigid inclusion with a Kapitza interface cannot been handled with the present approach and could probably solved by adapting the accelerated scheme based on the polarization [25]. It must be noted that the Kapitza interface can be interpreted as a third thin phase with very low conductivity (see for instance [13]), as consequence, it is not really surprising that the rate of convergence is not significantly affected. However, a dual approach which consists to formulate the iterative scheme with the flux and the resistivity (inverse of the conductivity) is not divergent since the resistivity in the third phase tends to infinity, this has been shown in [28]. Note also that the primal approach based on the gradient of temperature delivers an upper bound for 
the effective conductivity but it is then not possible to obtain the lower bound by the dual formulation.

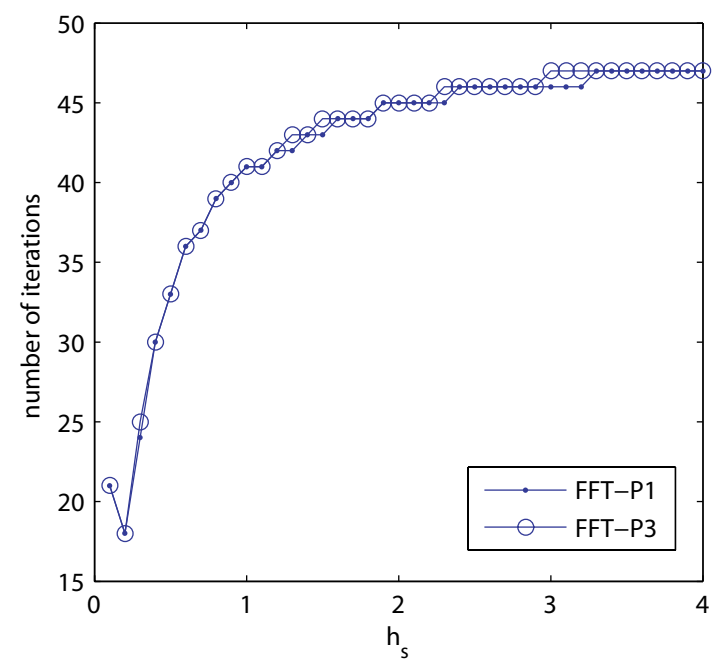

Fig. 4. Number of iterations at convergence as function $h_{s}$ for $k_{1}=1, k_{2}=10$ and $R=0.25$.

\subsection{Comparison with Finite Element solutions}

The effective conductivity of the composite is represented on Fig. 5 as function of the fiber radius $R$ for $k_{1}=1$ and for various values of $h_{s}$ and $k_{2}$. The results obtained with FFT-P1 and FFT-P3 are compared with Finite Element (FE) solutions. The FE solution is computed with 15098 triangular elements, linear interpolation functions in each element and by doubling the temperature at each nodes located at the interface in order to introduce the discontinuity. One observes a good agreement between the FFT and the FE solutions. It is however observed a slight discrepancy of the FFT-P1 solution when the inclusion radius is close to $R=0.5$. When $h_{s}=0.5$ we observe that the two curves decrease, reach a minimum and then increase. This illustrates a competition between the interface and the inclusion effects on the effective conductivity. Indeed, the inclusion, which is more conductive that the matrix, increases the effective conductivity while the discontinuity at the interface decreases the effective conductivity. 

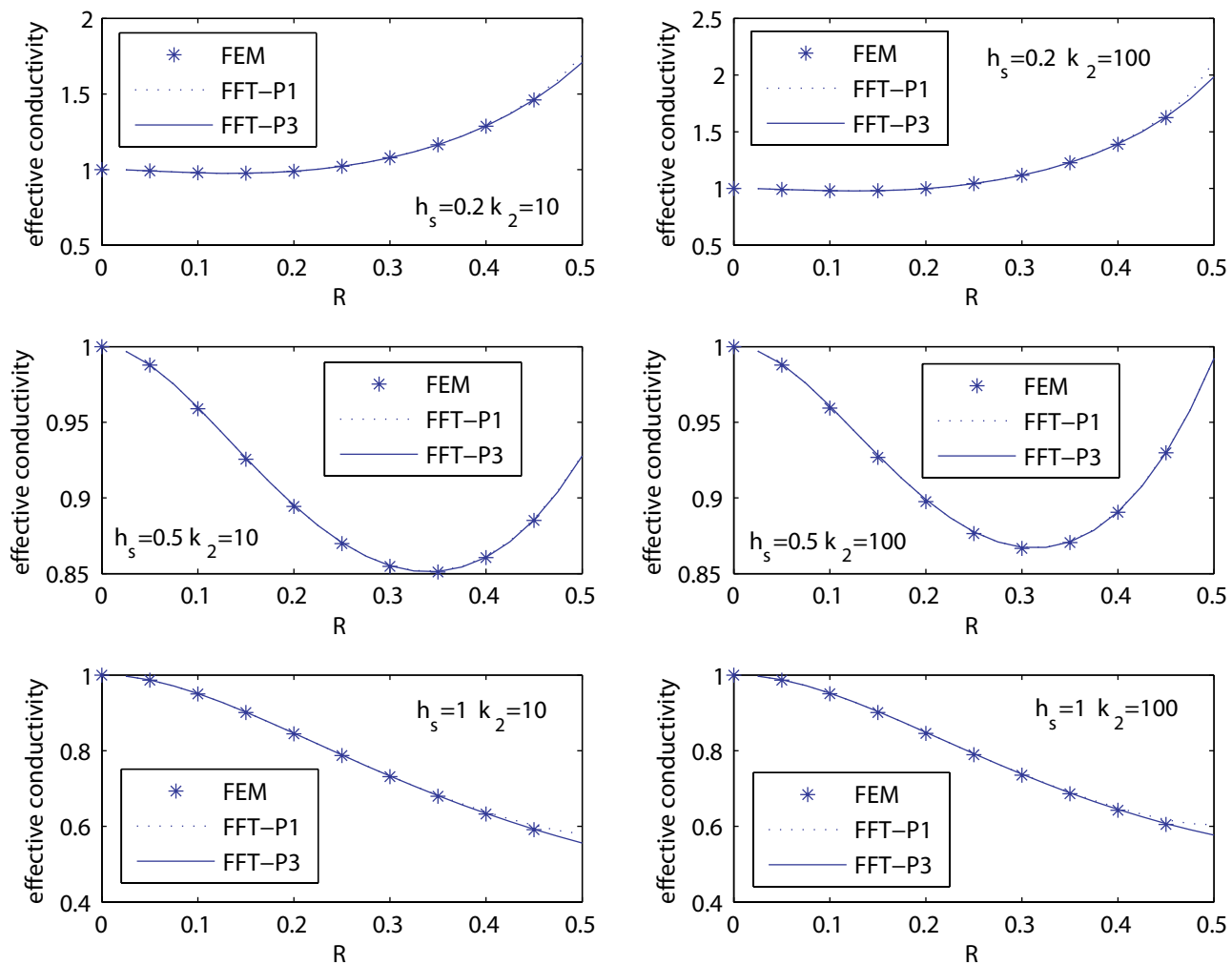

Fig. 5. Variations of the effective conductivity with the fiber radius $R$. Comparison between the FFT solutions with the FE one.

A qualitative comparison with the FE solutions is provided on tables 1 and 2 for the radius $R=0.2$ and $R=0.4$ respectively. For each value of the radius, we consider for the thermal resistance: $h_{s}=0.2, h_{s}=0.5$ and $h_{s}=1$ while the conductivity of the inclusion is $k_{2}=10$ and $k_{2}=100$. Considering the case $R=0.2$, the results obtained with FFT-P1, FFT-P3 and FFT-P5 are the same at a given value of the resolution excepted for $h_{s}=1$ and $k_{2}=100$ for which FFT-P3 and FFT-P5 give a slightly more refined estimation. For $R=0.2$, the FFT-P1 solution is accurate and the consideration of higher order polynomials is not required. In the case $R=0.4$, one observes a significant difference between FFT-P1 and FFT-P3. However the solutions computed with FFT-P3 and FFT-P5 are identical. This suggests that the use of third order polynomials is sufficient to reproduce the discontinuity at the interface. 


\begin{tabular}{|c|c|c|c|c|c|c|c|}
\cline { 2 - 8 } \multicolumn{2}{c|}{} & \multicolumn{2}{c|}{$h_{s}=0.2$} & \multicolumn{2}{c|}{$h_{s}=0.5$} & \multicolumn{2}{c|}{$h_{s}=1$} \\
\hline \multirow{3}{*}{$64 \times 64$} & 10 & 100 & 10 & 100 & 10 & 100 \\
\cline { 2 - 8 } & FFT-P1 & 0.9881 & 0.9988 & 0.8952 & 0.8989 & 0.8462 & 0.8486 \\
\cline { 2 - 8 } & FFT-P3 & 0.9881 & 0.9988 & 0.8952 & 0.8989 & 0.8462 & 0.8486 \\
\cline { 2 - 8 } & FFT-P5 & 0.9881 & 0.9988 & 0.8952 & 0.8989 & 0.8462 & 0.8486 \\
\hline \multirow{3}{*}{$128 \times 128$} & FFT-P1 & 0.9881 & 0.9988 & 0.8948 & 0.8983 & 0.8454 & 0.8471 \\
\cline { 2 - 8 } & FFT-P3 & 0.9881 & 0.9988 & 0.8948 & 0.8983 & 0.8454 & 0.8471 \\
\cline { 2 - 8 } & FFT-P5 & 0.9881 & 0.9988 & 0.8948 & 0.8983 & 0.8454 & 0.8471 \\
\hline \multirow{3}{*}{$256 \times 256$} & FFT-P1 & 0.9881 & 0.9988 & 0.8946 & 0.8980 & 0.8450 & 0.8464 \\
\cline { 2 - 8 } & FFT-P3 & 0.9881 & 0.9988 & 0.8946 & 0.8980 & 0.8449 & 0.8463 \\
\cline { 2 - 8 } & FFT-P5 & 0.9881 & 0.9988 & 0.8946 & 0.8980 & 0.8449 & 0.8463 \\
\hline \multirow{2}{*}{ FEM } & 0.9881 & 0.9987 & 0.8944 & 0.8976 & 0.8446 & 0.8456 \\
\hline
\end{tabular}

Table 1

Comparison of the effective conductivity computed with the FEM and the FFT for $R=0.2$

\begin{tabular}{|c|c|c|c|c|c|c|c|}
\cline { 2 - 8 } \multicolumn{2}{c|}{} & \multicolumn{2}{c|}{$h_{s}=0.2$} & \multicolumn{2}{c|}{$h_{s}=0.5$} & \multicolumn{2}{c|}{$h_{s}=1$} \\
\hline \multirow{4}{*}{ contrast= } & 10 & 100 & 10 & 100 & 10 & 100 \\
\hline \multirow{3}{*}{$128 \times 64$} & FFT-P1 & 1.2897 & 1.3958 & 0.8613 & 0.8912 & 0.6384 & 0.6493 \\
\cline { 2 - 8 } & FFT-P3 & 1.2880 & 1.3919 & 0.8607 & 0.8908 & 0.6342 & 0.6448 \\
\cline { 2 - 9 } & FFT-P5 & 1.2880 & 1.3919 & 0.8607 & 0.8908 & 0.6342 & 0.6448 \\
\hline \multirow{3}{*}{$256 \times 28$} & FFT-P1 & 1.2894 & 1.3944 & 0.8612 & 0.8911 & 0.6378 & 0.6482 \\
\cline { 2 - 9 } & FFT-P3 & 1.2876 & 1.3907 & 0.8606 & 0.8907 & 0.6336 & 0.6438 \\
\cline { 2 - 8 } & FFT-P5 & 1.2876 & 1.3907 & 0.8606 & 0.8907 & 0.6336 & 0.6438 \\
\hline & FFT-P1 & 1.2892 & 1.3937 & 0.8612 & 0.8910 & 0.6375 & 0.6476 \\
\cline { 2 - 8 } & FFT-P3 & 1.2874 & 1.3901 & 0.8606 & 0.8907 & 0.6333 & 0.6432 \\
\cline { 2 - 8 } & FFT-P5 & 1.2874 & 1.3901 & 0.8606 & 0.8907 & 0.6333 & 0.6432 \\
\hline \multicolumn{2}{|c|}{ FEM } & 1.2869 & 1.3890 & 0.8605 & 0.8905 & 0.6329 & 0.6426 \\
\hline
\end{tabular}

Table 2

Comparison of the effective conductivity computed with the FEM and the FFT for $R=0.4$

In order to assess the accuracy of the local fields, the jump of the temperature 
field across the interface is represented on figures 6 to 8 for $R=0.2, R=0.4$ and $R=0.49$ respectively. The jump distribution is represented as function of the angle coordinate $\theta$ which vary from 0 to $2 \pi$. The contrast is 100 and the thermal resistance of the interface is $h_{s}=1$. We compare FFT-P1 and FFTP3 solutions with the FE one. A very good agreement for the temperature jump distribution is observed for FFT-P1 and FFT-P3 solutions when the radius is $R=0.2$. Also for $R=0.4$ the FFT-P1 is still closed to the FE solution but the FFT-P3 one leads to a better approximation. For $R=0.49$, that corresponding to almost touching inclusions, the discrepancy between the FE and FFT-P1 solutions is more significant. Also, differences are observed between FFT-P3 and FE solutions. Obviously, the good comparisons between the FFT solutions and the FE one is due to cylindrical shape which has a very smooth interface. Comparisons would be provided for other inclusions shape to evaluate the accuracy of the method by considering, for example, the case of a rectangular inclusion shape.

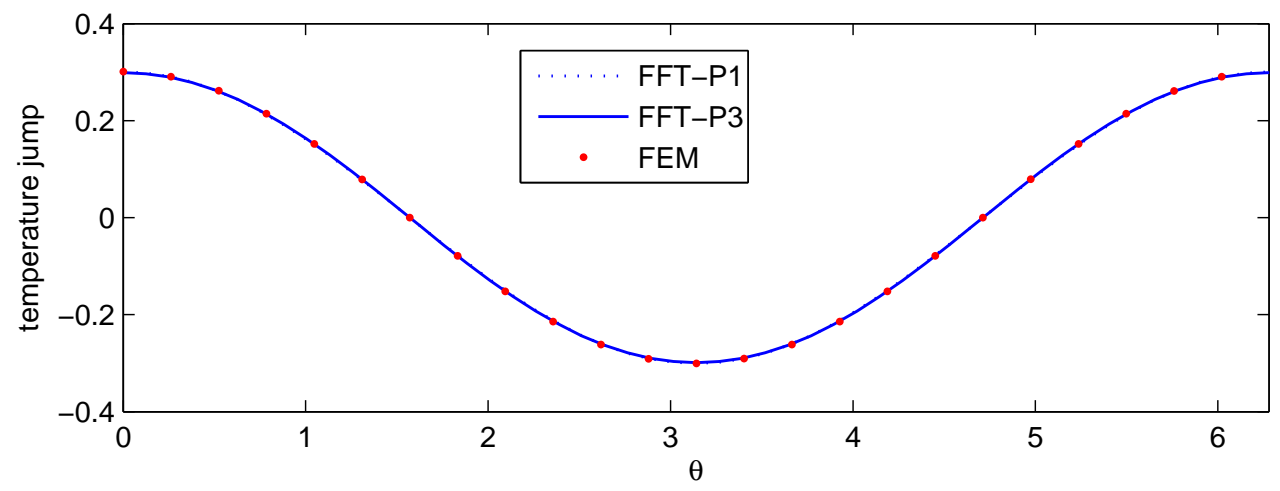

Fig. 6. Variations of the temperature jump as function of the angular coordinate $\theta$ for $R=0.2, h_{s}=1$ and a contrast of 100 .

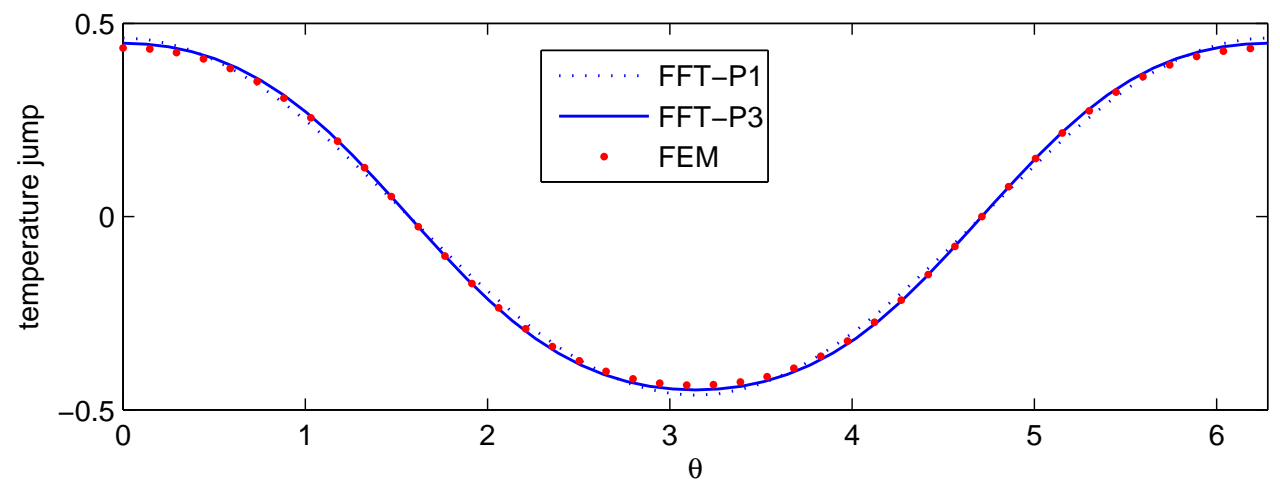

Fig. 7. Variations of the temperature jump as function of the angular coordinate $\theta$ for $R=0.4, h_{s}=1$ and a contrast of 100 . 


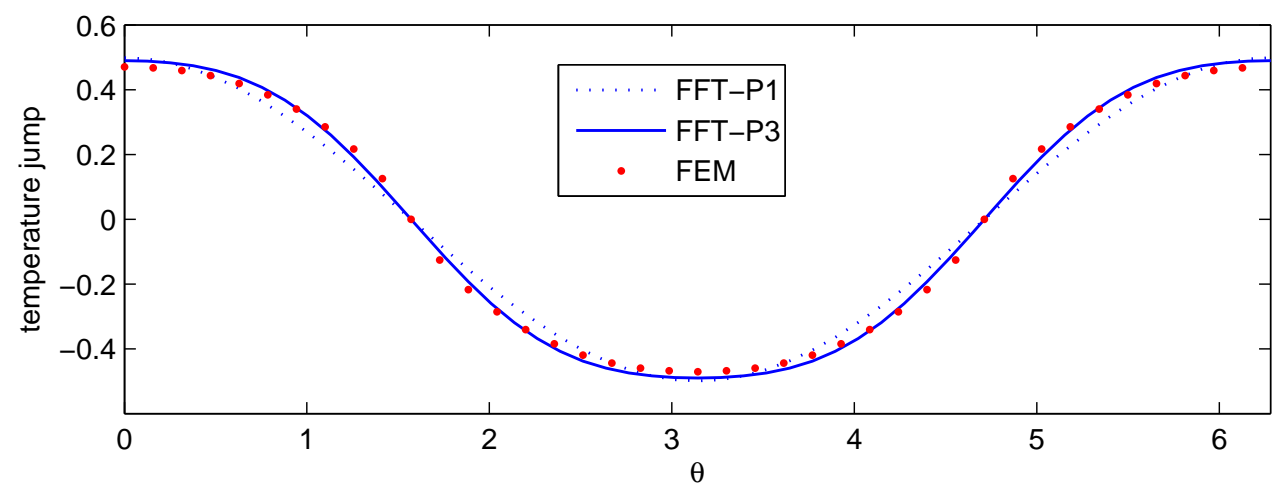

Fig. 8. Variations of the temperature jump as function of the angular coordinate $\theta$ for $R=0.49, h_{s}=1$ and a contrast of 100 .

\section{$5 \quad$ Unit cell with a population of fibers}

Consider now a unit cell containing $\mathcal{P}$ fibers of radius $R$ randomly distributed (see Fig. 9). The position of each fiber is defined by $\boldsymbol{X}^{(i)}$ for $i=1$..P . Since all the inclusions have the same radius, the coefficients $\mathcal{R}_{i j}$, given by Eq. (45), are the same for each fibers. Vectors $\boldsymbol{\pi}_{j}^{(i)}(\boldsymbol{\xi})$ depend on the position $\boldsymbol{X}^{(i)}$ and, by making use of the translation property of the Fourier transform, they are computed from the following relation:

$$
\boldsymbol{\pi}_{j}^{(i)}(\boldsymbol{\xi})=\boldsymbol{\pi}_{j}^{0}(\boldsymbol{\xi}) \exp \left(i \boldsymbol{\xi} \cdot \boldsymbol{X}^{(i)}\right)
$$

where $\boldsymbol{\pi}_{j}^{0}(\boldsymbol{\xi})$ with the superscript "0" is associated to a fiber located at $\boldsymbol{x}=0$ whose expressions for $j=1 . . \mathcal{Q}$ are given by Eq. (44).

As in the last example, the interpolation functions $\boldsymbol{w}_{3}(\boldsymbol{x}), \boldsymbol{w}_{8}(\boldsymbol{x})$ and $\boldsymbol{w}_{9}(\boldsymbol{x})$ are omitted since they do not introduce a discontinuity at the interface. All other interpolation functions are now considered in the computation of the linear operator $\boldsymbol{L}_{n, m}$. In this example, the interpolation functions $\boldsymbol{w}_{4}$ and $\boldsymbol{w}_{5}$ must kept due to the inclusion interactions. However, the results show that the influence of these two functions is negligible and could omitted. Then, only functions $w_{1}(\boldsymbol{x}), w_{2}(\boldsymbol{x}), w_{6}(\boldsymbol{x})$ and $w_{7}(\boldsymbol{x})$ are used. So, the total number of interpolation functions remains $\mathcal{Q}=4$. 


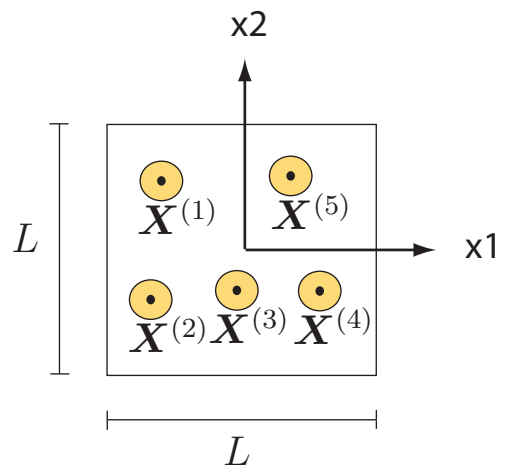

Fig. 9. Unit cell

The unit cells of the random microstructures are illustrated on Fig. 9. Four different microstructures are shown with $\mathcal{P}=30$ inclusions. The volume fraction of reinforcements is $\mathcal{P} \pi R^{2} / L^{2}=0.236$. In this example, we study the sizedependence of the effective thermal conductivity of the composite. The thermal conductivity of the matrix and the inclusion are $k_{1}=1 \mathrm{Wm}^{-1} \mathrm{~K}^{-1}, k_{2}=$ $10 \mathrm{Wm}^{-1} \mathrm{~K}^{-1}$ while the thermal resistance of the interface is $h_{s}=10^{-5} \mathrm{~m}^{2} \mathrm{~K} / \mathrm{W}$ (here we use some values considered in [15]). The calculations are performed with dimensionless parameters. A unit cell having the dimension 1 along each space directions is considered containing $\mathcal{P}$ inclusions with the dimensionless radius:

$$
R^{*}=\frac{R}{L}
$$

and the dimensionless Kapitza resistance:

$$
h_{s}^{*}=\frac{h_{s}}{k_{1} L}
$$

Eliminating $L$ in Eqs (52) and (53), one obtains for the dimensionless Kapitza resistance:

$$
h_{s}^{*}=\frac{h_{s} R^{*}}{k_{1} R}
$$

From a practical point of view, the size-dependency of the effective conductivity is studied by decreasing the radius of the inclusions $R$, and then, by increasing the dimensionless Kapitza resistance $h_{s}^{*}$. In this example, the radius $R$ of the inclusion vary from $0.02 \mu \mathrm{m}$ to $250 \mu \mathrm{m}$. The values of the effective conductivity for each occurrences are represented on Fig. 10 for $R=250 \mu \mathrm{m}$ and on Fig. 11 for $R=0.1 \mu \mathrm{m}$. The cumulated average value of the effective conductivity is also computed for each inclusion radius. 

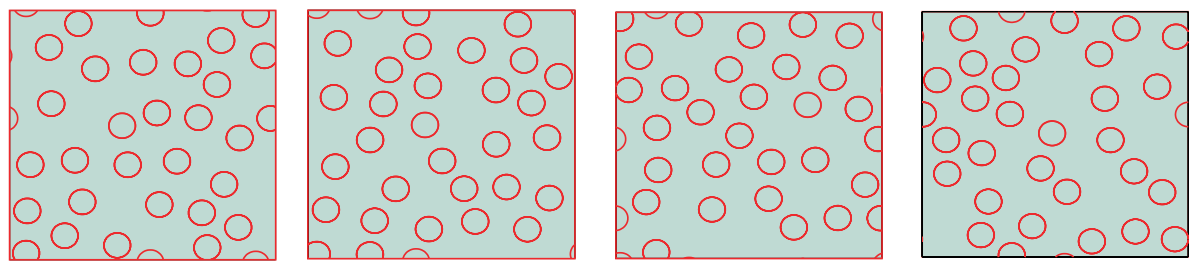

Fig. 10. Unit cells of random microstructures.

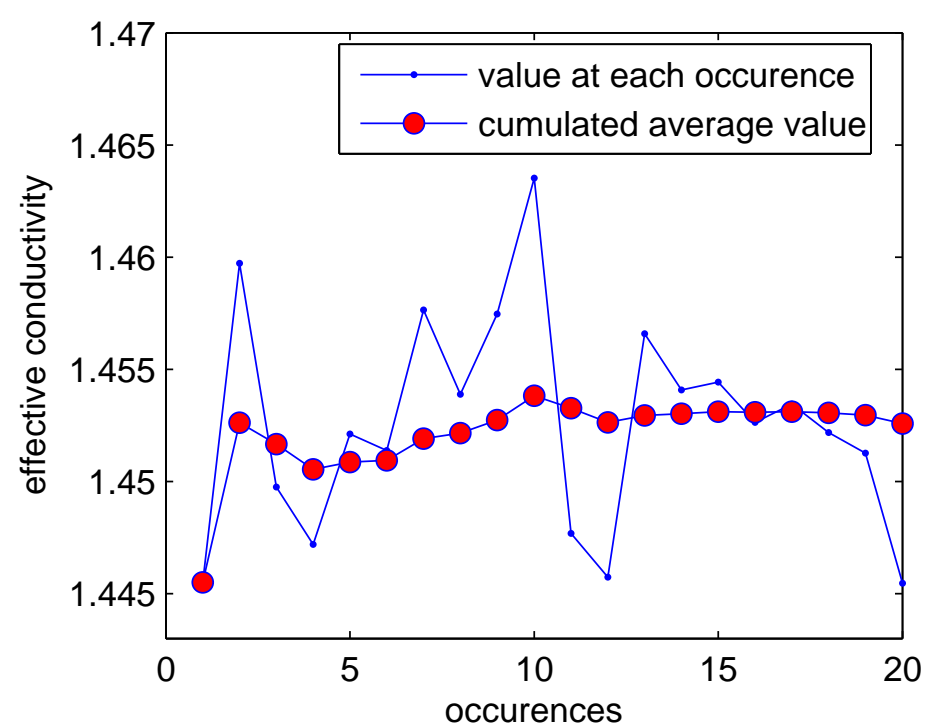

Fig. 11. Statistical convergence of the effective conductivity for $R=250 \mu m$

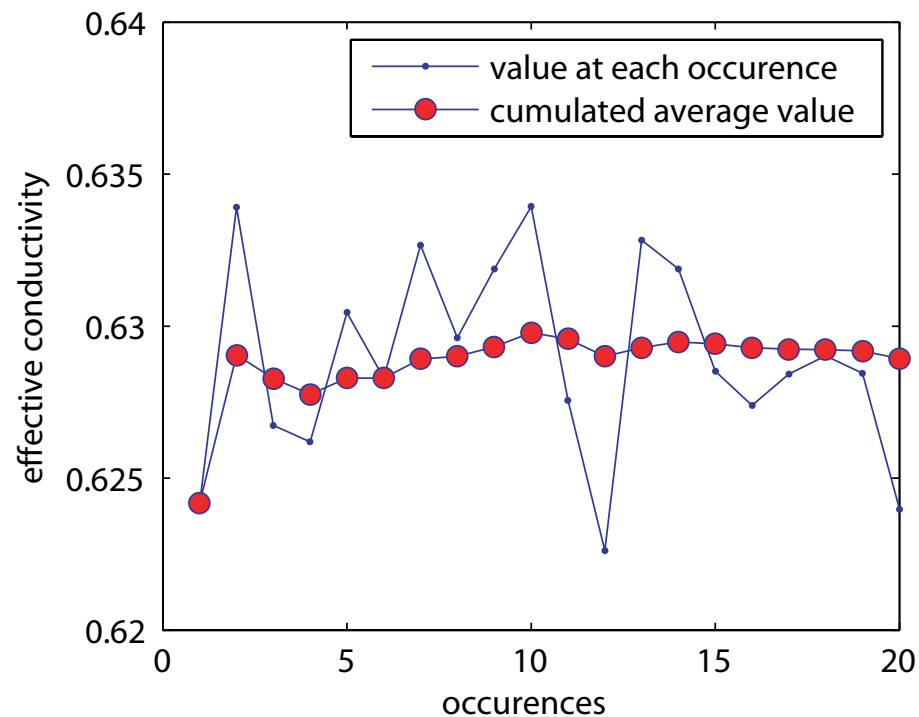

Fig. 12. Statistical convergence of the effective conductivity for $R=0.1 \mu \mathrm{m}$

On Table 3, we compare the cumulated average value of the effective con- 
ductivity obtained with FFT-P1 and FFT-P3 for 20 occurrences. It can be observed that solutions are very closed whatever the value of the inclusion radius $\mathrm{R}$. The maximum difference is observed for $R=0.02 \mu \mathrm{m}$ and the relative difference is $0.08 \%$.

\begin{tabular}{|c|c|c|c|c|c|c|c|}
\hline $\mathrm{R}($ in $\mu \mathrm{m})$ & 250 & 50 & 12.5 & 2.5 & 0.5 & 0.1 & 0.02 \\
\hline FFT-P1 & 1.4526 & 1.2993 & 1.0252 & 0.7541 & 0.6565 & 0.6336 & 0.6289 \\
\hline FFT-P3 & 1.4527 & 1.2994 & 1.0252 & 0.7543 & 0.6569 & 0.6341 & 0.6294 \\
\hline
\end{tabular}

Table 3

Cumulated average value of the effective conductivity obtained with 20 occurrences. Comparison between the FFT-P1 and FFT-P3 solutions.

The variations of the effective conductivity with the size of the inclusions are presented on Fig. 13. The discrete points corresponds to the FFT-P3 solutions. Also, analytic estimates of the size-dependent effective conductivity are provided on this figure: the dilute scheme, the Mori-Tanaka scheme and the self-consistent scheme (these estimates being derived in [15]). The size dependency with the inclusion radius is clearly observed. Also, it can be noticed that the numerical results obtained for this microstructure are very closed to the Mori-Tanaka estimate.

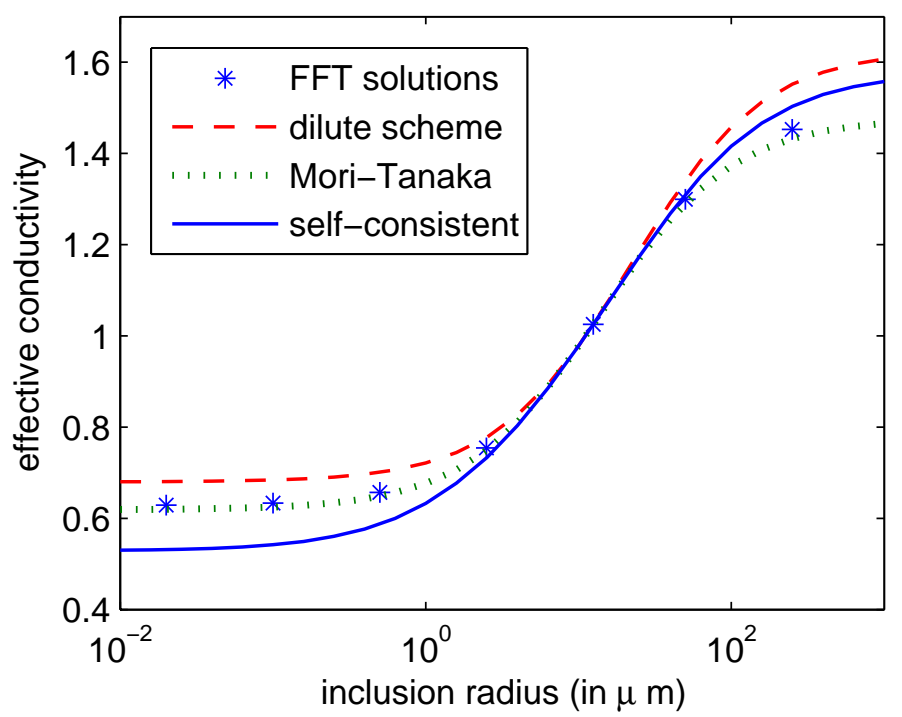

Fig. 13. Variations of the effective conductivity with the radius size for of the composites with random distribution of fibers.

The case of a unit cell containing 80 fibers which are different in size is now considered and illustrated on Fig. 14. The radii of the inclusions are randomly chosen in the range $R=[10 \mu m, 40 \mu m]$. The values of $k_{1}, k_{2}$ and $h_{s}$ remains 
unchanged. We still use the FFT-P3 approximation for computing the effective conductivity. The shape functions of the fibers are all different, they are computed and stored before the iteration process. The convergence of the effective conductivity with the number of wave vectors is provided on Fig. 15. The computation time are compared on Fig. 16 for: a unit cell is one inclusion (Fig. 1), a unit cell with 30 inclusions with the same radius (Fig. 10) and a unit cell with 80 inclusions with different radii. It is observed that the computation time linearly depends on the number of wave vectors $N$ in the log-log frame. Also, the computation time increases with the number of inclusions. The reason is that in the computation of $\boldsymbol{L}_{n, m}$, given by (34), an iteration over the total number of inclusions $\mathcal{P}$ is required.

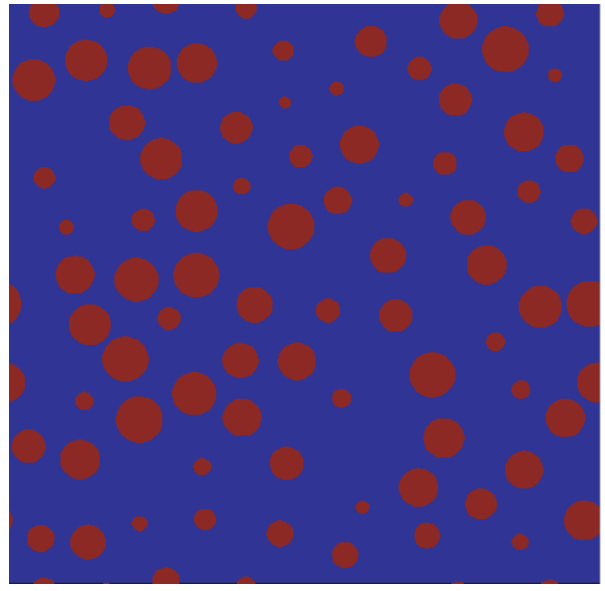

Fig. 14. Unit cell with 80 different inclusions in size.

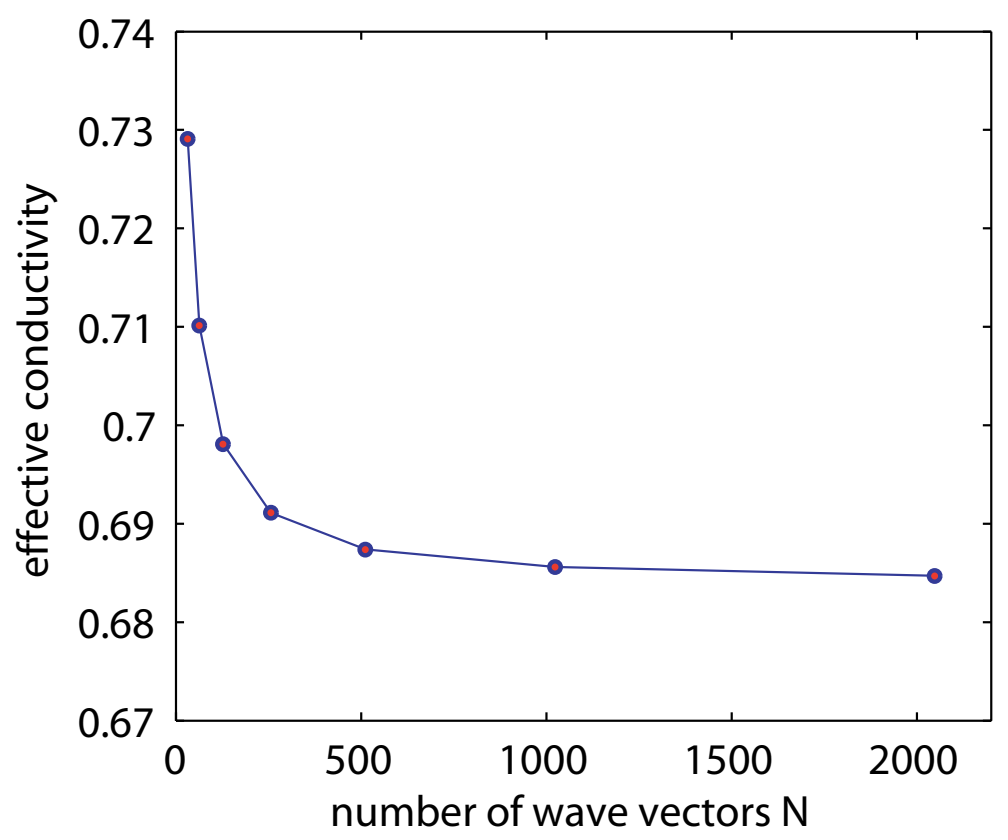

Fig. 15. Convergence of the effective conductivity with the number of wave vectors $N$ in the case of 80 different inclusions in size 


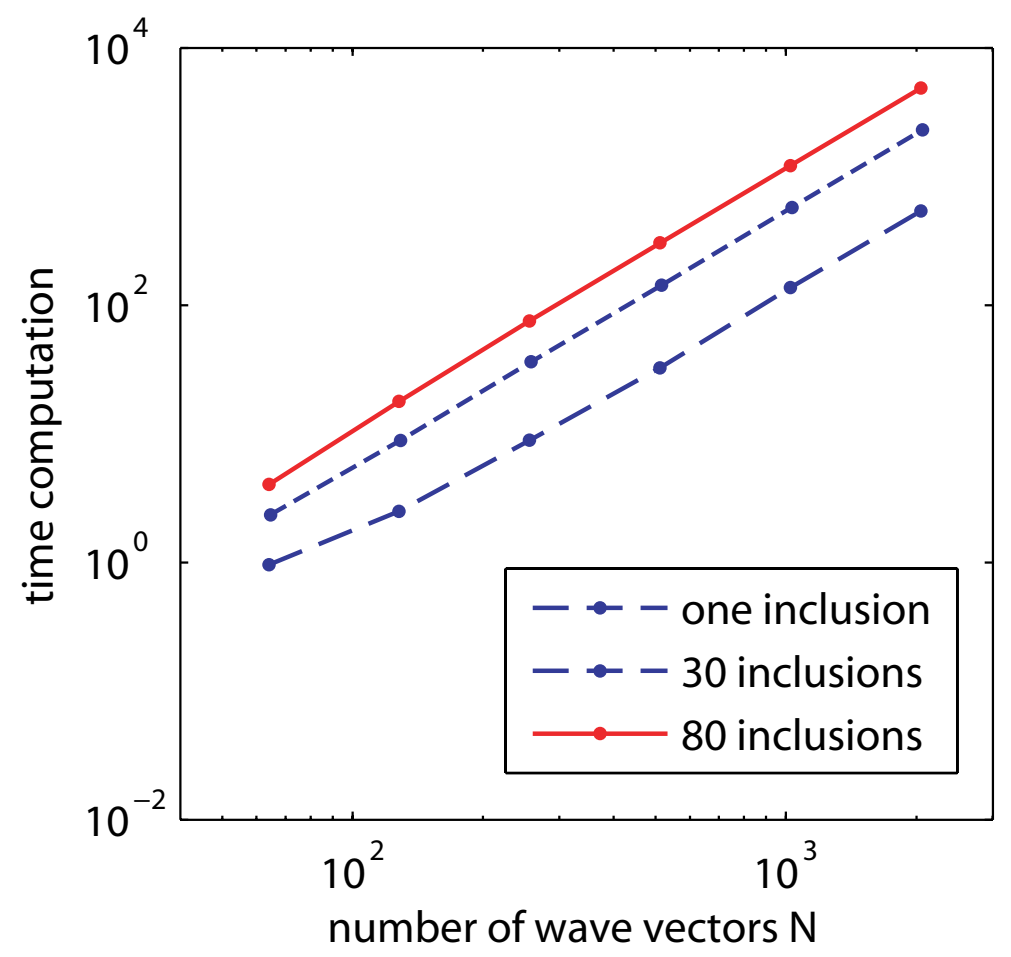

Fig. 16. Computation times required for the different type of microstructures.

\section{Conclusion}

In this paper we extend the method based on FFT to compute the effective conductivity of composites with imperfect interfaces. For this purpose, the Kapitza model has been considered at the interface between the matrix and the cylindrical inclusions. The Kapitza model involves a discontinuity of the temperature across the interface that is proportional to the flux acting on the interface, the latter remaining continuous across the interface. The principle of the approach is to consider, in a variational procedure, Fourier series and additional temperature fields which are null outside the inclusions. A representation of these functions with polynomial interpolation functions is proposed and the method is applied to fiber reinforced composites. The resulting iterative scheme is similar to that already derived by Moulinec and Suquet [27]: it uses the exact expression of the periodic Green tensor and the FFT algorithm to compute the Fourier transform and it inverse. The surface effects are captured by a modified conductivity tensor which account for the thermal resistance of the interface. The case of a composite with regularly distributed inclusions is considered and the rate of convergence of the algorithm is first studied. The results show that the convergence is not significantly modified as compared to the case of composites with perfect interfaces. In order to check the accuracy of the method, we provide comparisons with finite element so- 
lutions, still in the case of a regular array of fibers. Also, the accuracy of the FFT solution is investigated by comparing the distribution of the temperature jump across the interface with the FE solution. The results show that the enrichment with a third order polynomial is sufficient to obtain a good accuracy. Next, we apply the method for composites with random distribution of fibers and we compare the results with analytic solutions coming from the homogenization theory. The results show the ability of the method to account for the size-dependency of the effective conductivity of the composite with the inclusion radius. The application of the method to 3D problems and other inclusions shape is in progress. Particularly, for more complex geometries of the inclusions, the approach could surely be performed in tandem with the use of Level Set functions as already used by the XFEM method (see for instance [36]). The method could be also extend to other mechanical or physical problems involving surface discontinuities.

\section{References}

[1] Belytschko T., Black V. Elastic crack growth in finite elements with minimal remeshing. Int. J. Numer. Methods Eng.45(5):601-620, 1999.

[2] Benveniste Y., Miloh T. The effective conductivity of composites with imperfect thermal contact at constituent interfaces. Int. J. Engrg. Science. 24(9):1537-1552, 1986.

[3] Bonnet G. Effective properties of elastic periodic composite media with fibers. J. Mech. Phys. Solids. 55:881-899, 2007.

[4] Brisard S. and Dormieux L. FFT-based methods for the mechanics of composites: A general variational framework. Comput. Mater. Science. 49(3):663-671, 2010.

[5] Brisard S., Dormieux L., Kondo D. HashinÜShtrikman bounds on the bulk modulus of a nanocomposite with spherical inclusions and interface effects. Comp. Mater. Science. 48(3):589-596, 2010.

[6] Challis L.J. Kapitza resistance and acoustic transmission across boundaries at high frequencies. Journal of Physics C: Solid State Physics. 7(3):481-495, 1974.

[7] T. Chen, G.J. Dvorak. Fibrous nanocomposites with interface stress: Hill's and Levin's connections for effective moduli. Appl. Phys. Lett. 88:211912-1-3, 2006 .

[8] Duan L., Wang J., Huang Z.P., Karihaloo B.L. Size-dependent effective elastic constants of solids containing nano-inhomogeneities with interface stress. J. Mech. Phys. Solids. 53:1574-1596, 2005. 
[9] Dunn M.L., Taya M. The effective thermal conductivity of composites with coated reinforcement and the application to imperfect interfaces. J. Appl. Phys. 73:1711, 1993.

[10] Eyre D.J., Milton G.W. A fast numerical scheme for computing the response of composites using grid refinement. J. Phys. III. 6:41-47, 1999.

[11] Goland M., Reissner E. The stresses in cemented joints. J. Appl. Mech. 11:A17ÜA27, 1944.

[12] Gurtin M.E., Murdoch A.I. A continuum theory of elastic material surfaces. Arch. Rat. Mech. Anal. 57:291-323, 1975.

[13] Hashin Z. Thin interphase/imperfect interface in conduction. J. Appl. Physics. 89:2261, 2001.

[14] Hashin Z. Thin interphase/imperfect interface in elasticity with application to coated fiber composites. J. Mech. Phys. Solids. 50:2509-2537, 2002.

[15] Le-Quang H., He Q.-C. Variational principles and bounds for elastic inhomogeneous materials with coherent imperfect interfaces. Mechanics of Materials. 40:865-884, 2008.

[16] Le Quang H., He Q.-C., Bonnet G. Eshelby's tensor fields and effective conductivity of composites made of anisotropic phases with Kapitza's interface thermal resistance. Phil.l Mag. 91(25):3358-3392, 2011.

[17] Ly H.-B., Monchiet V., Grande D. Computation of permeability with Fast Fourier Transform from 3-D digital images of porous microstructures. Int. J. Num. Meth. Heat \& Fluid Flow. 26(5), 1328-1345, 2016.

[18] Jones, J.P., Whittier, J.S. Waves at a Vexible bonded interface. J. Appl. Mech. 34:905Ü909, 1967.

[19] Moës N., Dolbow J., Belytschko T. A finite element method for crack growth without remeshing. Int. J. Numer. Meth. Engrg. 46(1):131-156, 1999.

[20] Kapitza, P.L. The study of heat transfer in helium II. J. Phys. 4:181-210, 1941.

[21] Michel J. C., Moulinec H. and Suquet P. A computational scheme for linear and non-linear composites with arbitrary phase contrast. Int. J. Numer. Meth. Engng., 52:139-160, 2001.

[22] Milton G. W.

The Theory of Composites. Cambridge University Press, 2002.

[23] Moës N., Dolbow J., Belytschko T. A finite element method for crack growth without remeshing. Int. J. Numer. Methods Eng. 46(1):131-156, 1999.

[24] Monchiet V., Bonnet G., Lauriat G. A FFT-based method to compute the permeability induced by a Stokes slip flow through a porous medium. Comptes Rendus Mécanique. 337(4):192-197, 2009. 
[25] Monchiet V., Bonnet G. A polarization based FFT iterative scheme for computing the effective properties of elastic composites with arbitrary contrast. Int. J. Num. Meth. Engrg. 89(11):1419-1436, 2012.

[26] Monchiet V. Combining FFT methods and variational principles to compute bounds and estimates for the properties of elastic composites. Comput. Meth. Appl. Mech. Engrg. 283:454-473, 2015.

[27] Moulinec H. and Suquet P. A fast numerical method for computing the linear and nonlinear mechanical properties of composites. C. R. Acad. Sci. 318(11):1417-1423, 1994.

[28] Nguyen T.-K. Homogénéisation numérique des structures périodiques par transformée de Fourier : applications aux composites et aux milieux poreux. PhD thesis. University Paris-Est Marne-la-Vallée. 2010.

[29] Nguyen T.-K., Monchiet V., Bonnet G. A Fourier based numerical method for computing the dynamic permeability of porous media. European Journal of Mechanics B/fluids. 37:90-98, 2013.

[30] Nemat-Nasser S, Hori M. Micromechanics: Overall Properties of Heterogeneous Materials. North- Holland, Amsterdam, 1999.

[31] Povstenko Y.Z. Theoretical investigation of phenomena caused by heterogeneous surface tension in solids. J. Mech. Phys. Solids. 41:1499-1514, 1993.

[32] Sharma P., Ganti S. Size-Dependent EshelbyŠs Tensor for Embedded Nano-Inclusions Incorporating Surface/Interface Energies. Journal of Applied Mechanics. 71:663-671, 2004.

[33] Sukumar N., Chopp D.L., Moës N., Belytschko T. Modeling holes and inclusions by level sets in the extended finite-element method. Comput. Meth. Appl. Mech. Eng. 190:6183-6200, 2001.

[34] To V.-T., To Q.-D., Monchiet V. On the Inertia Effects on the Darcy Law: Numerical Implementation and Confrontation of Micromechanics-Based Approaches. Transport in Porous Media. 111(1):171-191, 2016.

[35] Torquato S., Rintoul M.D. Effect of the Interface on the Properties of Composite Media. Phys. Rev. Lett. 75:4067, 1995.

[36] Yvonnet J., Le-Quang H., He Q.-C. An XFEM/level set approach to modelling surface/interface effects and to computing the size-dependent effective properties of nanocomposites. Computational Mechanics. 42(1):119$131,2008$.

[37] Yvonnet J., He Q.-C., Toulemonde C. Numerical modelling of the effective conductivities of composites with arbitrarily shaped inclusions and highly conducting interface. Composites Science and Technology. 68(13): 2818-2825, 2008 . 
[38] Zhu Q.-Z., Gu S.-T., Yvonnet J., Shao J.-F., He Q.-C. Imperfect interfaces with XFEM: new developments in enrichment function. Int. J. Numer. Meth. Engng. 88:307Ü32, 2011.

\section{A Expressions of higher order polynomials}

Fourth order polynomials are:

$$
\begin{array}{r}
w_{10}(\boldsymbol{x})=x_{1}^{4}-6 x_{1}^{2} x_{2}^{2}+x_{2}^{4} \\
w_{11}(\boldsymbol{x})=x_{1} x_{2}\left(x_{2}^{2}-x_{1}^{2}\right) \\
w_{12}(\boldsymbol{x})=x_{1} x_{2}\left(x_{1}^{2}+x_{2}^{2}-R^{2}\right) \\
w_{13}(\boldsymbol{x})=\left(x_{1}^{2}+x_{2}^{2}-R^{2}\right)\left(3 x_{2}^{2}+3 x_{1}^{2}-R^{2}\right) \\
w_{14}(\boldsymbol{x})=\left(x_{2}^{2}-x_{1}^{2}\right)\left(x_{1}^{2}+x_{2}^{2}-R^{2}\right)
\end{array}
$$

Fifth order polynomials are:

$$
\begin{array}{r}
w_{15}(\boldsymbol{x})=x_{1}\left(x_{1}^{4}-10 x_{1}^{2} x_{2}^{2}+5 x 2^{4}\right) \\
w_{16}(\boldsymbol{x})=x_{2}\left(x_{2}^{4}-10 x_{1}^{2} x_{2}^{2}+5 x 1^{4}\right) \\
w_{17}(\boldsymbol{x})=x_{1}\left(x_{1}^{2}+x_{2}^{2}-R^{2}\right)\left(2 x_{2}^{2}+2 x_{1}^{2}-R^{2}\right) \\
w_{18}(\boldsymbol{x})=x_{2}\left(x_{1}^{2}+x_{2}^{2}-R^{2}\right)\left(2 x_{2}^{2}+2 x_{1}^{2}-R^{2}\right) \\
w_{19}(\boldsymbol{x})=x_{1}\left(x_{1}^{2}-3 x_{2}^{2}\right)\left(x_{1}^{2}+x_{2}^{2}-R^{2}\right) \\
w_{20}(\boldsymbol{x})=x_{2}\left(x_{2}^{2}-3 x_{1}^{2}\right)\left(x_{1}^{2}+x_{2}^{2}-R^{2}\right)
\end{array}
$$

